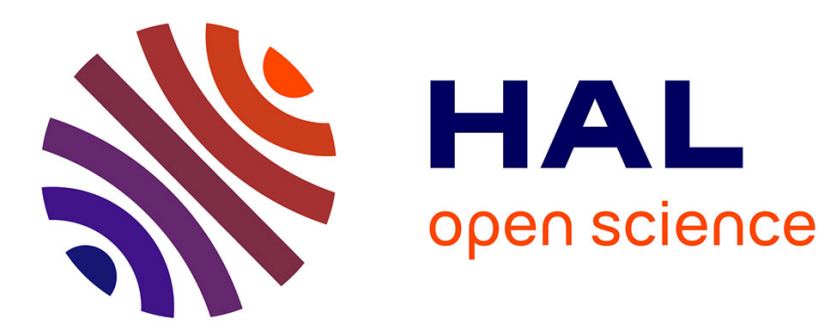

\title{
Anti-windup strategies for discrete-time switched systems subject to input saturation
}

Marc Jungers, Sophie Tarbouriech

\section{To cite this version:}

Marc Jungers, Sophie Tarbouriech. Anti-windup strategies for discrete-time switched systems subject to input saturation. International Journal of Control, 2016, 89 (5), pp.919-937. 10.1080/00207179.2015.1105384 . hal-01242540

\section{HAL Id: hal-01242540 \\ https://hal.science/hal-01242540}

Submitted on 25 Feb 2016

HAL is a multi-disciplinary open access archive for the deposit and dissemination of scientific research documents, whether they are published or not. The documents may come from teaching and research institutions in France or abroad, or from public or private research centers.
L'archive ouverte pluridisciplinaire HAL, est destinée au dépôt et à la diffusion de documents scientifiques de niveau recherche, publiés ou non, émanant des établissements d'enseignement et de recherche français ou étrangers, des laboratoires publics ou privés. 


\title{
Regular Paper
}

\section{Anti-Windup strategies for discrete-time switched systems subject to input saturation}

\author{
Marc Jungers $^{a, b *}$ and Sophie Tarbouriech ${ }^{c}$ \\ ${ }^{a}$ Université de Lorraine, CRAN, UMR 7039, 2 avenue de la forêt de Haye, Vandœuvre-lès-Nancy \\ Cedex, 54516, France; ${ }^{b}$ CNRS, CRAN, UMR 7039, France; ${ }^{c} C N R S$, LAAS, 7 avenue du Colonel Roche, \\ F-31400 Toulouse, France and Univ de Toulouse, LAAS, F-31400 Toulouse, France
}

(v3.5 released August 2008)

\begin{abstract}
This paper deals with the design of anti-windup compensator for discrete-time switched systems subject to input saturation. The cases of static and dynamic anti-windup controllers are addressed aiming at maximizing the estimate of the basin of attraction of the origin for the closed-loop system. Two aspects of the switching law are taken into account during the design: either it is arbitrary or it is a part of the complete control law. Theoretical conditions allowing to synthesize the anti-windup compensator are mainly described through linear matrix inequalities. Computational oriented conditions are then provided to solve convex optimization problems able to give a constructive solution.
\end{abstract}

Keywords: Switched systems; anti-windup; Lyapunov functions; saturation; deadzone; Lyapunov-(Metzler) inequalities.

\section{Introduction}

The stability and the stabilization of systems including saturations is an important field of research in control theory. It has been motivated by the fact that most real applications present limitations on the actuators and/or sensors for physical or safety reasons. The presence of a saturation may be a source of instability, or at least of only local stability, and implies frequently a reduction of the performances ( $\mathrm{Hu}$ and Lin 2001, Kapila and Grigoriadis 2002, Tarbouriech et al. 2007): for instance, the presence of a saturation may reduce the size of the basin of attraction of the origin.

Among the different techniques to cope with control of systems including saturations, the antiwindup strategy is one of the most popular. It consists of the introduction of control modifications in order to recover, as much as possible, the performance induced by a previous control design, which did not take into account the presence of the saturation. The literature about anti-windup strategy is rich as it can be seen in (Turner and Zaccarian 2006, Galeani et al. 2009, Tarbouriech et al. 2011, Zaccarian and Teel 2011) and references therein. It has been more particularly focused on continuous-time systems, even if some contributions on anti-windup control design for discretetime systems could be underlined, as for example (Gomes da Silva Jr. et al. 2001, Herrmann et al. 2003, Turner et al. 2003, Gomes da Silva Jr. and Tarbouriech 2006, Grimm et al. 2008).

On the other hand, a large range of complex systems, as for instance embedded ones, can be modeled as a hybrid dynamical system, exhibiting characteristics of both continuous-time and discrete-time dynamical systems (Lunze and Lamnabhi-Lagarrigue 2009). They can be modeled

\footnotetext{
*Corresponding author. Email: marc.jungers@univ-lorraine.fr
} 
as a state, which can flow on a flow set by verifying a differential inclusion and can jump on a jump set by verifying a difference inclusion (Goebel et al. 2012). An important class of hybrid systems consists of switched systems, that is a finite set of dynamics, associated with a switching rule indicating at each time the mode that is active (Liberzon 2003, van der Schaft and Schumacher 2000, Lunze and Lamnabhi-Lagarrigue 2009). Indeed, the state of a switched system satisfies a differential (or difference) equation, which is parametrized by the switching rule that can jump between the different indices of the modes (see, for instance, (Goebel et al. 2012, Section 1.4.4)). The switched systems can be distinguished by the nature of the switching rule, which can be - in function of the application - considered as a perturbation (properties should thus be ensured for all the admissible switching rules), or as a part of the control inputs. The issue of the stability and the stabilization of switched systems has induced a great number of contributions, because it is not intuitive (Liberzon and Morse 1999). For instance, a switched system, switching between stable modes, may be unstable. Several tools have been already proposed to investigate the stability of switched systems: multiple Lyapunov functions (Branicky 1998), Lie algebra conditions (Liberzon et al. 1999, Agrachev and Liberzon 2001, Shorten et al. 2007), common Lyapunov functions (Hante and Sigalotti 2011), switching Lyapunov functions (Daafouz, Riedinger et al. 2002), variational approach (Monovich and Margaliot 2011), joint spectral radius (Tsitsiklis and Blondel 1997) (see also (Ye et al. 1998, Sun and Ge 2005)). The difficulty of this issue increases when the switched system is subjected to constraints (Daafouz et al. 2013).

Recently both aspects (saturation and switching nature) inherent to realistic complex systems have been considered together. First of all, a link may be established naturally by formulating the saturation as a switch between piecewise linear models (Johansson 2002) or between the input and the associated deadzone (Yuan and Wu 2014). Without a reformulation of the saturation, the stability and stabilization of discrete-time switched systems with input saturation has been investigated in (Liu and Duan 2005) with a common Lyapunov function and afterward in (Ma et al. 2009) with a saturation-dependent Lyapunov function, by extending the contribution in (Cao and Lin 2003). The continuous-time case has been studied in (Ni and Cheng 2010) by using a minimum dwell time. Several contributions are focused on the uncertainties (Zhang et al. 2010) or the $\mathcal{L}_{2}$-gain performances (Zhang et al. 2011, Zhao and Zhao 2014). The early work on optimal control for linear hybrid systems subject to saturation (Schutter 2000) is also noteworthy. One can also mention (Benzaouia 2012), where the switched systems subject to input saturation are considered by using in particular Linear Differential Inclusions issued from (Hu and Lin 2001) to deal with such a limitation. In the case of discrete-time switching systems with a nonlinear mode satisfying a modal cone bounded sector condition, a new Lyapunov function depending on the modal nonlinearity has been provided in (Gonzaga et al. 2011, 2012a) when the switching law is arbitrary and in (Jungers et al. 2012, 2013) when the switching law is a part of the control. These results are valid for generic nonlinearity (not necessarily a saturation or a deadzone) and are based on the introduction of this kind of Lyapunov functions for the standard discrete-time Lur'e problem (Gonzaga et al. 2012b).

The anti-windup techniques and the switched formalism have been also gathered in the literature. For instance, it is emphasized in (Bruckner et al. 2010) (see also (Bruckner et al. 2013)) that even if the plant does not switch, a switching anti-windup compensator may improve the performances. Pionner contributions applying (switching) anti-windup techniques on switched systems have been provided in the literature. In the continuous-time framework several other tools have been considered: multiple Lyapunov functions (Lu and Lin 2009, 2010), piecewise quadratic Lyapunov functions (Mulder and Kothare 2000, Tiwari et al. 2007) or being focused on uncertainties. We may mention the following work on a particular class of switched systems (Shorten et al. 2009).

The results (Zhang et al. 2012, Zhang and Zhao 2012, Chen et al. 2012) considering the discretetime case, are more particularly focused on the presence of a model uncertainty or of an exogenous perturbative input, when the switching law is arbitrary. The class of static anti-windup controllers considered in these contributions, affects the state evolution equation of the compensator, de- 
signed with the linear system and not directly the output of the compensator. When the switching law can be chosen, the anti-windup compensators can be used to improve the performances of the closed-loop system, such as the size of the domain of attraction (Duan and Wu 2012, 2014, Li and Lin 2013).

Due to the fact that a few works about anti-windup strategy for switched systems including saturation in discrete-time have been published as mentioned above, the aim of the present paper is to bridge the gap left by the literature and then to provide a panel of results in this framework by proposing static and dynamic anti-windup controllers when the switched rule is arbitrary or a part of the control inputs.

The paper is organized as follows. Section 2 presents the problem statements and states the main tools which will be used in the sequel. Sections 3 and 4 provide respectively a static antiwindup controller and a dynamic output one design in the case of arbitrary switching law, which maximizes the size of the estimate of the basin of attraction. Sections 5 and 6 extend the results to the case where the switching law is a part of the control. Also refinements are given allowing a switching law design based only on the knowledge of the system output. Illustrations are presented in Section 7, whereas Section 8 ends the paper with concluding remarks.

Notation. $\mathbb{N}, \mathbb{R}, \mathbb{R}^{+}$are respectively the set of the natural integers, of the real numbers and of the nonnegative real numbers. For any vector $x \in \mathbb{R}^{n}, x \geq 0$ states that, $\forall \ell=1, \ldots, n$, its components $x_{(\ell)}$ are nonnegative. Also, for two vectors $x, y$ of $\mathbb{R}^{n}, x \geq y$ states that $x_{(\ell)}-y_{(\ell)} \geq 0$, $\forall \ell=1, \ldots, n$. $A_{(\ell)}$ and $A_{i,(\ell)}$ denote the $\ell$-th row of matrices $A$ and $A_{i}$, respectively. For two symmetric matrices, $A$ and $B, A>B$ means that $A-B$ is positive definite. $A^{\prime}$ and $A^{\perp}$ denote respectively the transpose and a basis of the null space of matrix $A$. $\operatorname{He}(A)=A+A^{\prime} . I_{m}\left(0_{m \times n}\right)$ is the $m$-order identity matrix $(m \times n$-order null matrix). $\star$ means the symmetric blocks in matrices. $\operatorname{diag}(A ; B)$ denotes the diagonal matrix whose diagonal blocks are formed by square matrices $A$ and $B$. For a symmetric positive-definite matrix $M \in \mathbb{R}^{n \times n}$, the ellipsoidal set $\mathcal{E}(M, \gamma)$ associated with $M=M^{\prime}>0$ and $\gamma>0$ is given by $\left\{x \in \mathbb{R}^{n} ; x^{\prime} M x \leq \gamma\right\}$ and the shortcut $\mathcal{E}(M)=\mathcal{E}(M, 1)$ is used.

\section{Problem statement}

Consider the switched discrete-time linear system consisting of $N$ modes

$$
\begin{aligned}
x(t+1) & =A_{\sigma(t)} x(t)+B_{\sigma(t)} u(t), \quad x(0)=x_{0}, \\
y(t) & =C_{\sigma(t)} x(t),
\end{aligned}
$$

where $x(t) \in \mathbb{R}^{n_{\mathrm{p}}}, u(t) \in \mathbb{R}^{m}$ and $y(t) \in \mathbb{R}^{p}$ are respectively the state, the input and the measured output of the system, at any time $t \in \mathbb{N}$. The mapping $\sigma: \mathbb{N} \mapsto \mathcal{I}=\{1, \cdots, N\}$ is called the switching rule and indicates at each time $t \in \mathbb{N}$ the unique active mode among $\left\{\left(A_{i}, B_{i}, C_{i}\right)\right\}_{i \in \mathcal{I}}$. It is assumed that a switching $n_{\mathrm{c}}$-order dynamic output controller is given as follows

$$
\begin{aligned}
x_{\mathrm{c}}(t+1) & =A_{\mathrm{c}, \sigma(t)} x_{\mathrm{c}}(t)+B_{\mathrm{c}, \sigma(t)} u_{\mathrm{c}}(t), \\
v_{\mathrm{c}}(t) & =C_{\mathrm{c}, \sigma(t)} x_{\mathrm{c}}(t)+D_{\mathrm{c}, \sigma(t)} u_{\mathrm{c}}(t),
\end{aligned}
$$

where $x_{\mathrm{c}}(t) \in \mathbb{R}^{n_{\mathrm{c}}}, u_{\mathrm{c}}(t)=y(t) \in \mathbb{R}^{p}$ and $v_{\mathrm{c}}(t) \in \mathbb{R}^{m}$ are respectively the state, the input and the output of the controller.

The properties of the controller given by (3)-(4) depend on two distinct and conflicting frameworks related to the switching law $\sigma$. They are gathered in the two following conflicting Assumptions 2.1 and 2.2 .

Assumption 2.1: The switching law is arbitrary and not a priori known, but its current value is assumed available. The controller (3)-(4) is then assumed to stabilize the closed-loop system (1) 
for any possible switching rule in the absence of control saturation, that is when $u(t)=v_{\mathrm{c}}(t)$.

Assumption 2.2: The switching law is a part of the control inputs of the system. It is then assumed that there exists a particular switching law $\sigma$ which stabilizes the closed-loop system consisting of the system (1) and of the controller (3)-(4) in the absence of control saturation, that is when $u(t)=v_{\mathrm{c}}(t)$.

It should be underlined that, in the last case (Assumption 2.2), the stability of the closed-loop system is not assumed for any switching law, but only for a few of them. The synthesis of the controller (3)-(4) may also take into account performance requirements in addition to stability aspects, as for instance in (Daafouz, Millérioux et al. 2002, Daafouz and Bernussou 2002, Daafouz et al. 2003, Melin et al. 2011).

Suppose now that the input $u(t)$ of the system is subject to magnitude limitations defined by

$$
\operatorname{sat}(u(t))_{(\ell)}=\operatorname{sat}\left(u_{(\ell)}(t)\right)=\operatorname{sign}\left(u_{(\ell)}(t)\right) \min \left(\rho_{(\ell)},\left|u_{(\ell)}(t)\right|\right), \quad \forall \ell \in\{1, \cdots, m\},
$$

where $\rho \in \mathbb{R}^{m}$ is fixed and verifies $\rho_{(\ell)}>0, \forall \ell \in\{1, \cdots, m\}$. The decentralized deadzone nonlinearity, dually associated with the saturation, is defined as

$$
\Psi(v)=\operatorname{sat}(v)-v, \quad \forall v \in \mathbb{R}^{m}
$$

To reduce the degradation of performance requirements due to the saturation of the input $u(t)$, anti-windup terms, denoted $y_{\mathrm{aw}, x}(t) \in \mathbb{R}^{n_{\mathrm{c}}}$ and $y_{\mathrm{aw}, v}(t) \in \mathbb{R}^{m}$ may be added to the controller (Tarbouriech et al. 2011), (Zaccarian and Teel 2011). In such a case, the whole system could be rewritten as

$$
\begin{aligned}
x(t+1) & =A_{\sigma(t)} x(t)+B_{\sigma(t)} \operatorname{sat}\left(v_{\mathrm{c}}(t)\right), \\
y(t) & =C_{\sigma(t)} x(t), \\
x_{\mathrm{c}}(t+1) & =A_{\mathrm{c}, \sigma(t)} x_{\mathrm{c}}(t)+B_{\mathrm{c}, \sigma(t)} C_{\sigma(t)} x(t)+y_{\mathrm{aw}, x}(t), \\
v_{\mathrm{c}}(t) & =C_{\mathrm{c}, \sigma(t)} x_{\mathrm{c}}(t)+D_{\mathrm{c}, \sigma(t)} C_{\sigma(t)} x(t)+y_{\mathrm{aw}, v}(t) .
\end{aligned}
$$

For a sake of clarity, we define the extended state

$$
\xi(t)=\left(\begin{array}{c}
x(t) \\
x_{\mathrm{c}}(t)
\end{array}\right) \in \mathbb{R}^{n}
$$

with $n=n_{\mathrm{p}}+n_{\mathrm{c}}$, the extended anti-windup term

$$
y_{\mathrm{aw}}(t)=\left(\begin{array}{l}
y_{\mathrm{aw}, x}(t) \\
y_{\mathrm{aw}, v}(t)
\end{array}\right) \in \mathbb{R}^{n_{\mathrm{c}}+m}
$$

and the extended matrices, $\forall i \in \mathcal{I}$,

$$
\begin{gathered}
\boldsymbol{A}_{i}=\left[\begin{array}{cc}
A_{i}+B_{i} D_{\mathrm{c}, i} C_{i} & B_{i} C_{\mathrm{c}, i} \\
B_{\mathrm{c}, i} C_{i} & A_{\mathrm{c}, i}
\end{array}\right] \in \mathbb{R}^{n \times n} ; \quad \boldsymbol{B}_{i}=\left[\begin{array}{c}
B_{i} \\
0_{n_{\mathrm{c}} \times m}
\end{array}\right] \in \mathbb{R}^{n \times m} ; \\
\boldsymbol{R}_{i}=\left[\begin{array}{cc}
0_{n_{\mathrm{p}} \times n_{\mathrm{c}}} & B_{i} \\
I_{n_{\mathrm{c}}} & 0_{n_{\mathrm{c}} \times m}
\end{array}\right] \in \mathbb{R}^{n \times\left(n_{\mathrm{c}}+m\right)} ; \quad \boldsymbol{K}_{i}=\left[D_{\mathrm{c}, i} C_{i} C_{\mathrm{c}, i}\right] \in \mathbb{R}^{m \times n} ; \\
\boldsymbol{F}=\left[0_{m \times n_{\mathrm{c}}} I_{m}\right] \in \mathbb{R}^{m \times\left(n_{\mathrm{c}}+m\right)} .
\end{gathered}
$$


The system can be reformulated into the compact form

$$
\begin{aligned}
\xi(t+1) & =\boldsymbol{A}_{\sigma(t)} \xi(t)+\boldsymbol{B}_{\sigma(t)} \Psi\left(v_{\mathrm{c}}(t)\right)+\boldsymbol{R}_{\sigma(t)} y_{\mathrm{aw}}(t), \\
v_{\mathrm{c}}(t) & =\boldsymbol{K}_{\sigma(t)} \xi(t)+\boldsymbol{F} y_{\mathrm{aw}}(t) .
\end{aligned}
$$

Notice that matrix $\boldsymbol{F}$, defined by (15) allows an extraction of the vector $y_{\text {aw }}(t)$ and is thus independent of the switching rule $\sigma$.

The following definitions will be common for the sequel of the paper.

Definition 2.3: The basin of attraction of the system (7)-(10), denoted $\mathcal{B}_{\mathrm{a}}$ is defined as the set of all $\xi_{0} \in \mathbb{R}^{n}$, such that the corresponding trajectories starting from any $\xi(0)=\xi_{0}$ converge asymptotically to the origin.

The notion of basin of attraction being delicate to cope with for non-linear systems, we introduce the following definition of region of asymptotic stability, allowing more conveniently to deal with basin of attraction estimates.

Definition 2.4: A set $\mathcal{R} \subset \mathcal{B}_{\text {a }}$, containing the origin in its interior, is said to be a region of asymptotic stability for the system (16) if the trajectories associated with any $\xi_{0} \in \mathcal{R}$ converge asymptotically to the origin.

The notion of trajectories appearing in Definitions 2.3 and 2.4 should be understood with respect to the choice of Assumption 2.1 or 2.2.

Among all the possible switched anti-windup compensators, we will study the static ones

$$
\begin{aligned}
& y_{\text {aw }, x}(t)=E_{\text {aw }, x, \sigma(t)} \Psi\left(v_{\mathrm{c}}(t)\right), \\
& y_{\text {aw }, v}(t)=E_{\text {aw }, v, \sigma(t)} \Psi\left(v_{\mathrm{c}}(t)\right),
\end{aligned}
$$

and dynamic ones, with the anti-windup compensator state $x_{\text {aw }}(t) \in \mathbb{R}^{n_{\text {aw }}}$,

$$
\begin{aligned}
x_{\mathrm{aw}}(t+1) & =A_{\mathrm{aw}, \sigma(t)} x_{\mathrm{aw}}(t)+B_{\mathrm{aw}, \sigma(t)} \Psi\left(v_{\mathrm{c}}(t)\right), \\
y_{\mathrm{aw}, x}(t) & =C_{\mathrm{aw}, x, \sigma(t)} x_{\mathrm{aw}}(t)+D_{\mathrm{aw}, x, \sigma(t)} \Psi\left(v_{\mathrm{c}}(t)\right), \\
y_{\mathrm{aw}, v}(t) & =C_{\mathrm{aw}, v, \sigma(t)} x_{\mathrm{aw}}(t)+D_{\mathrm{aw}, v, \sigma(t)} \Psi\left(v_{\mathrm{c}}(t)\right) .
\end{aligned}
$$

For a sake of clarity, the following notation are introduced: $\boldsymbol{E}_{\mathrm{aw}, i}=\left[\begin{array}{c}E_{\mathrm{aw}, x, i} \\ E_{\mathrm{aw}, v, i}\end{array}\right] \in \mathbb{R}^{\left(m+n_{\mathrm{c}}\right) \times m}$; $\boldsymbol{C}_{\mathrm{aw}, i}=\left[\begin{array}{c}C_{\mathrm{aw}, x, i} \\ C_{\mathrm{aw}, v, i}\end{array}\right] \in \mathbb{R}^{\left(n_{\mathrm{c}}+m\right) \times n_{\mathrm{aw}}}$ and finally $\boldsymbol{D}_{\mathrm{aw}, i}=\left[\begin{array}{c}D_{\mathrm{aw}, x, i} \\ D_{\mathrm{aw}, v, i}\end{array}\right] \in \mathbb{R}^{\left(n_{\mathrm{c}}+m\right) \times m}$.

We can list here the problems investigated in this paper:

Problem 2.5: Given the system (7)-(10), determine the anti-windup gain matrices $\boldsymbol{E}_{\mathrm{aw}, i}$, $i \in \mathcal{I}$ of the static anti-windup controller (18)-(19) leading to the largest estimate of the basin of attraction for the closed-loop system, under Assumption 2.1.

Problem 2.6: Given the system (7)-(10), determine the anti-windup gain matrices $A_{\mathrm{aw}, i}$, $B_{\text {aw }, i}, \boldsymbol{C}_{\text {aw }, i}$ and $\boldsymbol{D}_{\text {aw }, i},(i \in \mathcal{I})$ of the dynamic full order $\left(n_{\text {aw }}=n\right)$ anti-windup controller (20)(22) leading to the largest estimate of the basin of attraction for the closed-loop system, under Assumption 2.1.

Problem 2.7: Given the system (7)-(10), determine the anti-windup gain matrices $\boldsymbol{E}_{\text {aw }, i}, i \in \mathcal{I}$ of the static anti-windup controller (18)-(19) and conjointly the switching law $\sigma(t)$ leading to the largest estimate of the basin of attraction for the closed-loop system, under Assumption 2.2.

Problem 2.8: Given the system (7)-(10), determine the anti-windup gain matrices $A_{\mathrm{aw}, i}, B_{\mathrm{aw}, i}$, $\boldsymbol{C}_{\mathrm{aw}, i}$ and $\boldsymbol{D}_{\mathrm{aw}, i},(i \in \mathcal{I})$ of the dynamic full order $\left(n_{\mathrm{aw}}=n\right)$ anti-windup controller (20)-(22) 
and conjointly the switching law $\sigma(t)$ leading to the largest estimate of the basin of attraction for the closed-loop system, under Assumption 2.2.

Remark 1: It is noteworthy that these problems are not completely independent. For instance, a solution to Problem 2.5, that is matrices $\boldsymbol{E}_{\text {aw }, i}$, induces a particular solution to Problem 2.6 by imposing $A_{\mathrm{aw}, i}=0_{n_{\mathrm{aw}}}, B_{\mathrm{aw}, i}=0_{n_{\mathrm{aw}} \times m}$ and $C_{\mathrm{aw}, i}=0_{\left(n_{\mathrm{c}}+m\right) \times n_{\mathrm{aw}}}$ and $\boldsymbol{D}_{\mathrm{aw}, i}=\boldsymbol{E}_{\mathrm{aw}, i}$. The same relation occurs between Problems 2.7 and 2.8.

Each following section is related to solution to one of the problems described above, exploiting Lyapunov arguments and generalized sector conditions adapted to the switched systems.

\section{Static anti-windup controller for arbitrary switching law}

This section is devoted to solving Problem 2.5. The selected anti-windup controller is given by (18)-(19). This implies that the closed-loop system reads

$$
\begin{aligned}
\xi(t+1) & =\boldsymbol{A}_{\sigma(t)} \xi(t)+\left(\boldsymbol{B}_{\sigma(t)}+\boldsymbol{R}_{\sigma(t)} \boldsymbol{E}_{\mathrm{aw}, \sigma(t)}\right) \Psi\left(v_{\mathrm{c}}(t)\right), \\
v_{\mathrm{c}}(t) & =\boldsymbol{K}_{\sigma(t)} \xi(t)+\boldsymbol{F} \boldsymbol{E}_{\mathrm{aw}, \sigma(t)} \Psi\left(v_{\mathrm{c}}(t)\right) .
\end{aligned}
$$

Remark 2: Under Assumption 2.1, the controller (3)-(4) is such that all the trajectories starting from any initial condition $\xi(0) \in \mathbb{R}^{n}$ and which are solution to the difference inclusion $\xi(t+1) \in$ $\left\{\boldsymbol{A}_{i} \xi(t), \forall i \in \mathcal{I}\right\}$ converge to the origin. This difference inclusion is then called asymptotically stable (see (Molchanov and Pyatnitskiy 1989, Gurvits 1995, Daimond and Opoitsev 2001, Sun and Ge 2011)).

Remark 3: A particular attention should be paid to the well posedness of the implicit relation (24). Roughly speaking, for each $\xi(t)$, we can compute one and only one value $v_{\mathrm{c}}(t)$ satisfying the implicit relation (24). Due to the piecewise affine definition of the deadzone $\Psi\left(v_{\mathrm{c}}(t)\right)$ and the mode dependency, the implicit relation (24) can be rewritten as affine equations $\Gamma_{j} v_{\mathrm{c}}(t)+\mu_{j}(t)=0, j \in\left\{1, \cdots, N \times 3^{m}\right\}$, with $\Gamma_{j} \in \mathbb{R}^{m \times m}$ and $\mu_{j} \in \mathbb{R}^{m}$. We will verify a posteriori that the matrices $\Gamma_{j}$ are nonsingular, for any $j \in\left\{1, \cdots, N \times 3^{m}\right\}$, in order to ensure the well posedness of relation (24) (see for instance (Zaccarian and Teel 2011, Chapter 2, page 38) for more details). Notice that among this set of matrices, there are the matrices $\left(I_{m}+\boldsymbol{F} \boldsymbol{E}_{\mathrm{aw}, i}\right)$, $\forall i \in \mathcal{I}$.

Let us define the following set, which is necessary to associate the deadzone with a generalized sector condition. For a given set of matrices $H_{i} \in \mathbb{R}^{m \times n}, i \in \mathcal{I}$, one defines

$$
\mathcal{S}\left(\left\{H_{i}\right\}_{i \in \mathcal{I}}, \rho\right)=\left\{\xi \in \mathbb{R}^{n}:-\rho \leq H_{i} \xi \leq \rho, \forall i \in \mathcal{I}\right\} .
$$

Lemma 3.1: Consider matrices $G_{i} \in \mathbb{R}^{m \times n}, i \in \mathcal{I}$. If the vector $\xi$ is an element of $\mathcal{S}\left(\left\{G_{i}\right\}_{i \in \mathcal{I}}, \rho\right)$, then, the control law that is given by (24) satisfies the following sector condition

$$
\Psi^{\prime}\left(v_{c}\right) U_{i}\left[\operatorname{sat}\left(v_{c}\right)+G_{i} \xi\right] \leq 0,
$$

for any diagonal positive definite matrix $U_{i} \in \mathbb{R}^{m \times m}, \forall i \in \mathcal{I}$.

Proof The proof is straightforward from Lemma 1 in (Tarbouriech et al. 2006).

Remark 4: Because $v_{\mathrm{c}}$ depends on $\Psi\left(v_{\mathrm{c}}\right)$ by the definition (24), it is more suitable to consider the equivalent sector condition to (26), (see for instance (Hu et al. 2005, 2008) and (Tarbouriech et al. 2011, Chapter 7))

$$
\Psi^{\prime}\left(v_{\mathrm{c}}\right) U_{i}\left[\Psi\left(v_{\mathrm{c}}\right)+v_{\mathrm{c}}+G_{i} \xi\right] \leq 0 .
$$


Let us consider a switched quadratic function defined by

$$
V(\xi, i)=\xi^{\prime} P_{i} \xi, \quad \forall(\xi, i) \in \mathbb{R}^{n} \times \mathcal{I},
$$

with $\left\{P_{i}\right\}_{i \in \mathcal{I}}$ a set of symmetric and positive definite $n \times n$-matrices and the following candidate Lyapunov function:

$$
V_{\max }(\xi)=\max _{i \in \mathcal{I}} V(\xi, i), \quad \forall \xi \in \mathbb{R}^{n} .
$$

Let us define, also, the unitary level set of the Lyapunov function (29), which will be used, in the sequel, to estimate the basin of attraction of system (23). Consider the set

$$
L_{V_{\max }}=\left\{\xi \in \mathbb{R}^{n}: V_{\max }(\xi) \leq 1\right\}
$$

The level set $L_{V_{\max }}$ could be reformulated as indicated in the following lemma, (Hu 2007).

Lemma 3.2: $\quad$ The level set $L_{V_{\max }}$ defined by (30) verifies

$$
L_{V_{\max }}=\bigcap_{i \in \mathcal{I}} \mathcal{E}\left(P_{i}\right)
$$

Proof $\xi \in L_{V_{\max }}$ is equivalent to $V(\xi, i) \leq 1, \forall i \in \mathcal{I}$, that is $\xi \in \mathcal{E}\left(P_{i}\right), \forall i \in \mathcal{I}$, which is equivalent to $\xi \in \bigcap_{i \in \mathcal{I}} \mathcal{E}\left(P_{i}\right)$.

The following theorem offers a local stability condition for Problem 2.5.

Theorem 3.3: If there exists a set of symmetric positive definite matrices $W_{i} \in \mathbb{R}^{n \times n}$, matrices $Y_{i} \in \mathbb{R}^{m \times n}$, matrices $Z_{i} \in \mathbb{R}^{\left(m+n_{c}\right) \times m}$, and diagonal positive definite matrices $S_{i} \in \mathbb{R}^{m \times m}, i \in \mathcal{I}$ satisfying the LMIs

$$
\begin{gathered}
{\left[\begin{array}{ccc}
W_{j} & \star & \star \\
W_{i} \boldsymbol{A}_{i}^{\prime} & W_{i} & \star \\
\left(S_{i} \boldsymbol{B}_{i}^{\prime}+Z_{i}^{\prime} \boldsymbol{R}_{i}^{\prime}\right) & \left(\boldsymbol{K}_{i} W_{i}+Y_{i}\right) & \left(2 S_{i}+\boldsymbol{F} Z_{i}+Z_{i}^{\prime} \boldsymbol{F}^{\prime}\right)
\end{array}\right]>0, \quad \forall(i, j) \in \mathcal{I}^{2} ;} \\
{\left[\begin{array}{cc}
W_{i} & \star \\
Y_{i,(\ell)} & \rho_{(\ell)}^{2}
\end{array}\right]>0, \quad \forall(i, \ell) \in \mathcal{I} \times\{1, \cdots, m\},}
\end{gathered}
$$

then for the anti-windup gain $\boldsymbol{E}_{\text {aw,i }}=Z_{i} S_{i}^{-1}$, the level set $L_{V_{\max }}$ is a region of exponential stability for system (23), with $P_{i}=W_{i}^{-1}$.

Proof First of all, the coefficients $\rho_{(\ell)},(\ell \in\{1, \cdots, m\})$ being positive constants, a Schur complement (Boyd et al. 1994) could be applied to the inequality (33) and implies

$$
W_{i} \geq \frac{1}{\rho_{(\ell)}^{2}} Y_{i,(\ell)}^{\prime} Y_{i,(\ell)}
$$

By pre- and post-multiplying this inequality by $W_{i}^{-1}$ and by using the changes of variables $P_{i}=W_{i}^{-1}, G_{i}=Y_{i} W_{i}^{-1}(i \in \mathcal{I})$, we have

$$
P_{i} \geq \frac{1}{\rho_{(\ell)}^{2}} G_{i,(\ell)}^{\prime} G_{i,(\ell)}
$$


This implies that $L_{V_{\max }} \subset \mathcal{S}\left(\left\{G_{i}\right\}_{i \in \mathcal{I}}, \rho\right)$. Then from Lemma 3.1, it follows that the sector condition (26) is verified in the set $L_{V_{\max }}$.

By assuming the feasibility of inequality (32), the matrix $W_{j}$ is invertible and allows to apply a Schur complement and leads to

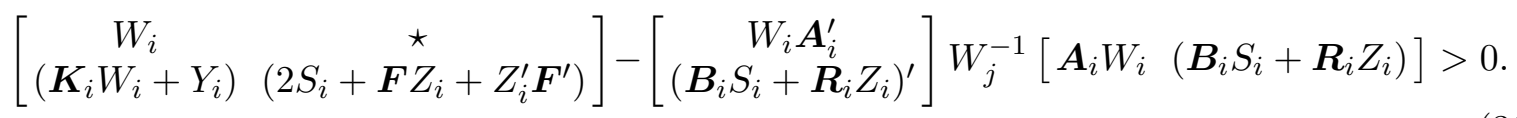

By using the changes of variables $U_{i}=S_{i}^{-1}, P_{i}=W_{i}^{-1}$ and $\boldsymbol{E}_{\text {aw }, i}=Z_{i} S_{i}^{-1},(i \in \mathcal{I})$, in addition of pre- and post-multiplying the inequality (36) by $\left[\begin{array}{cc}P_{i} & 0 \\ 0 & U_{i}\end{array}\right]$, one obtains

$$
\begin{aligned}
& {\left[\begin{array}{cc}
P_{i} & \star \\
\left(U_{i} \boldsymbol{K}_{i}+U_{i} G_{i}\right) & \left(2 U_{i}+U_{i} \boldsymbol{F} \boldsymbol{E}_{\mathrm{aw}, i}^{\star}+\boldsymbol{E}_{\mathrm{aw}, i}^{\prime} \boldsymbol{F}^{\prime} U_{i}\right)
\end{array}\right]} \\
& -\left[\begin{array}{c}
\boldsymbol{A}_{i}^{\prime} \\
\left(\boldsymbol{B}_{i}+\boldsymbol{R}_{i} \boldsymbol{E}_{\mathrm{aw}, i}\right)^{\prime}
\end{array}\right] P_{j}\left[\boldsymbol{A}_{i}\left(\boldsymbol{B}_{i}+\boldsymbol{R}_{i} \boldsymbol{E}_{\mathrm{aw}, i}\right)\right]>0 .
\end{aligned}
$$

By pre- and post-multiplying the inequality (37) respectively by $\left(\xi^{\prime}(t) \Psi^{\prime}\left(v_{\mathrm{c}}(t)\right)\right) \neq 0$ and its transpose, we have, by noting $i=\sigma(t)$ and $j=\sigma(t+1)$,

$$
V(\xi(t+1), \sigma(t+1))-V(\xi(t), \sigma(t))<2 \Psi^{\prime}\left(v_{\mathrm{c}}(t)\right) U_{\sigma(t)}\left[\Psi\left(v_{\mathrm{c}}(t)\right)+v_{\mathrm{c}}(t)+G_{\sigma(t)} \xi(t)\right] .
$$

By applying Lemma 3.1, we have for each $\xi(t) \in L_{V_{\max }}$, the stability condition

$$
V(\xi(t+1), \sigma(t+1))-V(\xi(t), \sigma(t)) \leq 0 .
$$

The inequality (37) being strict, for $\xi(t) \neq 0$, the stability condition (39) is thus strict, ensuring the stability of the origin in $L_{V_{\max }}$. The closed-loop system being an autonomous linear switched system, the origin in $L_{V_{\max }}$ is exponentially stable (Daafouz, Riedinger et al. 2002).

Theorem 3.3 provides sufficient conditions for the exponential stability in $L_{V_{\max }}$. In order to give a complete answer to Problem 2.5, one can build the following optimization problem, which is a way to maximize $L_{V_{\max }}$, the estimate of the basin of attraction.

Optimization problem 3.4

$$
\begin{gathered}
\min _{W_{i}, Y_{i}, Z_{i}, S_{i}, \beta} \beta \\
\text { subject to }(32)-(33) \text { and } \\
{\left[\begin{array}{cc}
\beta I_{n} & I_{n} \\
I_{n} & W_{i}
\end{array}\right]>0_{2 n}, \quad \forall i \in \mathcal{I} .}
\end{gathered}
$$

By using a Schur complement, the inequality (40) becomes

$$
\beta I_{n}>W_{i}^{-1}=P_{i}
$$

which induces the inclusion $\mathcal{E}\left(\beta I_{n}\right) \subset L_{V_{\max }}$, that is the ball of radius $\beta^{-\frac{1}{2}}$ is included into the estimate $L_{V_{\max }}$ of the basin of attraction $\mathcal{B}_{a}$.

Remark 5: It is noteworthy that only one scalar $\beta$ is considered in Optimization Problem 3.4 and in the LMI (40), because the aim is to increase the size of the intersection of the ellipsoids 
$\mathcal{E}\left(P_{i}\right), \forall i \in \mathcal{I}$. Of course the choice of the cost function to minimize is not unique. Other conventions may be possible, for instance, $\sum_{i \in \mathcal{I}} \operatorname{Tr}\left(P_{i}\right)$. A discussion about how to define the size of ellipsoids may be found in (Durieu et al. 1996) and (Tarbouriech et al. 2011).

\section{Dynamic anti-windup controller for arbitrary switching law}

This section copes with Problem 2.6, that is with a dynamic anti-windup controller described by (20)-(22). The state $x_{\text {aw }}(t)$ should be added to the extended state vector $\xi(t)$, in order to obtain the closed-loop compact form:

$$
\begin{aligned}
\eta(t+1) & =\left(\mathbb{A}_{\sigma(t)}+\mathbb{T}_{\sigma(t)} \mathbb{d}_{\sigma(t)} \mathbb{C}\right) \eta(t)+\left(\mathbb{B}_{\sigma(t)}+\mathbb{T}_{\sigma(t)} \mathbb{L}_{\sigma(t)}\right) \Psi\left(v_{\mathrm{c}}(t)\right), \\
v_{\mathrm{c}}(t) & =\left(\mathbb{K}_{\sigma(t)}+\mathbb{F} \mathbb{J}_{\sigma(t)} \mathbb{C}\right) \eta(t)+\mathbb{F} \mathbb{L}_{\sigma(t)} \Psi\left(v_{\mathrm{c}}(t)\right)
\end{aligned}
$$

with

$$
\eta(t)=\left(\begin{array}{c}
\xi(t) \\
x_{\mathrm{aw}}(t)
\end{array}\right) \in \mathbb{R}^{\hat{n}}, \quad \hat{n}=n+n_{\mathrm{aw}}
$$

and

$$
\begin{gathered}
\mathbb{A}_{i}=\left[\begin{array}{cc}
\boldsymbol{A}_{i} & 0_{n \times n_{\mathrm{aw}}} \\
0_{n_{\mathrm{aw}} \times n} & 0_{n_{\mathrm{aw}}}
\end{array}\right] ; \mathbb{C}=\left[0_{n_{\mathrm{aw}} \times n} I_{n_{\mathrm{aw}}}\right] ; \mathbb{F}=\left[0_{m \times n_{\mathrm{aw}}} \boldsymbol{F}\right] ; \\
\mathbb{T}_{i}=\left[\begin{array}{cc}
0_{n \times n_{\mathrm{aw}}} & \boldsymbol{R}_{i} \\
I_{n_{\mathrm{aw}}} & 0_{n_{\mathrm{aw}} \times\left(n_{\mathrm{c}}+m\right)}
\end{array}\right] ; \mathbb{B}_{i}=\left[\begin{array}{c}
\boldsymbol{B}_{i} \\
0_{n_{\mathrm{aw}} \times m}
\end{array}\right] ; \mathbb{K}_{i}=\left[\boldsymbol{K}_{i} 0_{m \times n_{\mathrm{aw}}}\right]
\end{gathered}
$$

and the variables

$$
\mathbb{L}_{i}=\left[\begin{array}{c}
B_{\mathrm{aw}, i} \\
D_{\mathrm{aw}, i}
\end{array}\right] \in \mathbb{R}^{\left(n_{\mathrm{aw}}+n_{\mathrm{c}}+m\right) \times m} ; \quad \mathbb{J}_{i}=\left[\begin{array}{c}
A_{\mathrm{aw}, i} \\
C_{\mathrm{aw}, i}
\end{array}\right] \in \mathbb{R}^{\left(n_{\mathrm{aw}}+n_{\mathrm{c}}+m\right) \times n_{\mathrm{aw}}} .
$$

Let us define the following set necessary to associate the deadzone with a generalized sector condition. For a given set of matrices $\mathbb{H}_{i} \in \mathbb{R}^{m \times \hat{n}}, i \in \mathcal{I}$, one defines

$$
\mathcal{S}\left(\left\{\mathbb{H}_{i}\right\}_{i \in \mathcal{I}}, \rho\right)=\left\{\eta \in \mathbb{R}^{\hat{n}} ;-\rho \leq \mathbb{H}_{i} \eta \leq \rho, \forall i \in \mathcal{I}\right\}
$$

Lemma 4.1: Consider matrices $\mathbb{G}_{i} \in \mathbb{R}^{m \times \hat{n}}, i \in \mathcal{I}$. If the vector $\eta$ is an element of $\mathcal{S}\left(\left\{\mathbb{G}_{i}\right\}_{i \in \mathcal{I}}, \rho\right)$, then, the control law given by (43) satisfies the following sector condition

$$
\Psi^{\prime}\left(v_{c}\right) \mathbb{U}_{i}\left[\operatorname{sat}\left(v_{c}\right)+\mathbb{G}_{i} \eta\right] \leq 0
$$

for any diagonal positive definite matrix $\mathbb{U}_{i} \in \mathbb{R}^{m \times m}, \forall i \in \mathcal{I}$, or equivalently

$$
\Psi^{\prime}\left(v_{c}\right) \mathbb{U}_{i}\left[\Psi\left(v_{c}\right)+v_{c}+\mathbb{G}_{i} \eta\right] \leq 0 .
$$

The switched quadratic function associated with the closed-loop system (42) is denoted

$$
\mathbb{V}(\eta, i)=\eta^{\prime} \mathbb{P}_{i} \eta, \quad \forall(\eta, i) \in \mathbb{R}^{\hat{n}} \times \mathcal{I},
$$


and induces the Lyapunov function

$$
\mathbb{V}_{\max }(\eta)=\max _{i \in \mathcal{I}} \mathbb{V}(\eta, i), \quad \forall \eta \in \mathbb{R}^{\hat{n}}
$$

The following theorem establishes sufficient conditions for the local exponential stability related to the closed-loop system (42).

Theorem 4.2: If there exists a set of symmetric positive definite matrices $\mathbb{P}_{i} \in \mathbb{R}^{\hat{n} \times \hat{n}}$, matrices $\mathbb{Q}_{i} \in \mathbb{R}^{m \times \hat{n}}, \mathbb{X}_{i} \in \mathbb{R}^{m \times m}, \mathbb{Y}_{i} \in \mathbb{R}^{\left(n_{\text {aw }}+n_{c}+m\right) \times n_{\text {aw }}}, \mathbb{Z}_{i} \in \mathbb{R}^{\left(n_{\text {aw }}+n_{c}+m\right) \times m}, \mathbb{M}_{i} \in$ $\mathbb{R}^{\left(n_{a w}+n_{c}+m\right) \times\left(n_{a w}+n_{c}+m\right)}$ and $\mathbb{N}_{i} \in \mathbb{R}^{\left(n_{a w}+n_{c}+m\right) \times\left(n_{a w}+n_{c}+m\right)}$, diagonal positive definite matrices $\mathbb{U}_{i} \in \mathbb{R}^{m \times m},(i \in \mathcal{I})$ such that, $\forall(i, j) \in \mathcal{I}^{2}$,

$$
\begin{array}{r}
{\left[\begin{array}{c}
\mathbb{A}_{i}^{\prime} \\
\mathbb{B}_{i}^{\prime} \\
\mathbb{T}_{i}^{\prime} \\
\mathbb{T}_{i}^{\prime} \\
0
\end{array}\right] \mathbb{P}_{j}\left[\begin{array}{c}
\mathbb{A}_{i}^{\prime} \\
\mathbb{B}_{i}^{\prime} \\
\mathbb{T}_{i}^{\prime} \\
\mathbb{T}_{i}^{\prime} \\
0
\end{array}\right]^{\prime}-\left[\begin{array}{c}
I_{\hat{n}} \\
0 \\
0 \\
0 \\
0
\end{array}\right] \mathbb{P}_{i}\left[\begin{array}{c}
I_{\hat{n}} \\
0 \\
0 \\
0 \\
0
\end{array}\right]^{\prime}-H e\left(\left[\begin{array}{c}
0 \\
I_{m} \\
0 \\
0 \\
0
\end{array}\right] \mathbb{U}_{i}\left[\begin{array}{c}
\mathbb{K}_{i}^{\prime} \\
I_{m} \\
\mathbb{F}^{\prime} \\
\mathbb{F}^{\prime} \\
I_{m}
\end{array}\right]\right)} \\
+H e\left(\left[\begin{array}{ccccc}
0 & 0 & 0 & 0 & 0 \\
0 & 0 & 0 & 0 & 0 \\
\mathbb{Y}_{i} \mathbb{C} & 0 & -\mathbb{M}_{i} & 0 & 0 \\
0 & \mathbb{Z}_{i} & 0 & -\mathbb{N}_{i} & 0 \\
\mathbb{Q}_{i} & 0 & 0 & 0 & -\mathbb{X}_{i}
\end{array}\right]\right)<0,
\end{array}
$$

and

$$
\left[\begin{array}{ccc}
\mathbb{P}_{i} & \star & \star \\
0_{1 \times \hat{n}} & \rho_{(\ell)}^{2} & \star \\
-\mathbb{Q}_{i} & I_{m,(\ell)}^{\prime} & H e\left(\mathbb{X}_{i}\right)
\end{array}\right]>0, \quad \forall(i, \ell) \in \mathcal{I} \times\{1, \cdots, m\}
$$

then for the dynamic anti-windup compensator defined by $\mathbb{L}_{i}=\mathbb{N}_{i}^{-1} \mathbb{Z}_{i}$ and $\mathbb{J}_{i}=\mathbb{M}_{i}^{-1} \mathbb{Y}_{i},(i \in \mathcal{I})$, the level set $L_{\mathbb{V}_{\max }}$ is a region of exponential stability for the system (42).

Proof The matrix $\mathbb{P}_{j}$ being symmetric positive definite, the two last diagonal blocks of the inequality (53) imply that the matrices $\mathbb{M}_{i}$ and $\mathbb{N}_{i}$ are invertible. Due to the last diagonal blocks of LMIs (54), $\mathbb{X}_{i}$ are also invertible. By assuming the change of variables $\mathbb{J}_{i}=\mathbb{M}_{i}^{-1} \mathbb{Y}_{i}$ and $\mathbb{L}_{i}=\mathbb{N}_{i}^{-1} \mathbb{Z}_{i}, \mathbb{G}_{i}=\mathbb{X}_{i}^{-1} \mathbb{Q}_{i}$, the inequality (53) can be rewritten as

$$
\begin{aligned}
{\left[\begin{array}{c}
\mathbb{A}_{i}^{\prime} \\
\mathbb{B}_{i}^{\prime} \\
\mathbb{T}_{i}^{\prime} \\
\mathbb{T}_{i}^{\prime} \\
0
\end{array}\right] \mathbb{P}_{j}\left[\begin{array}{c}
\mathbb{A}_{i}^{\prime} \\
\mathbb{B}_{i}^{\prime} \\
\mathbb{T}_{i}^{\prime} \\
\mathbb{T}_{i}^{\prime} \\
0
\end{array}\right]^{\prime} } & -\left[\begin{array}{c}
I_{\hat{n}} \\
0 \\
0 \\
0 \\
0
\end{array}\right] \mathbb{P}_{i}\left[\begin{array}{c}
I_{\hat{n}} \\
0 \\
0 \\
0 \\
0
\end{array}\right]^{\prime}-\operatorname{He}\left(\left[\begin{array}{c}
0 \\
I_{m} \\
0 \\
0 \\
0
\end{array}\right] \mathbb{U}_{i}\left[\begin{array}{c}
\mathbb{K}_{i}^{\prime} \\
I_{m} \\
\mathbb{F}^{\prime} \\
\mathbb{F}^{\prime} \\
I_{m}
\end{array}\right]\right) \\
& +\mathrm{He}\left(\left[\begin{array}{ccc}
0 & 0 & 0 \\
0 & 0 & 0 \\
\mathbb{M}_{i} & 0 & 0 \\
0 & \mathbb{N}_{i} & 0 \\
0 & 0 & \mathbb{X}_{i}
\end{array}\right]\left[\begin{array}{ccccc}
\mathbb{J}_{i} \mathbb{C} & 0 & -I_{n_{\mathrm{aw}}+n_{\mathrm{c}}+m} & 0 & 0 \\
0 & \mathbb{L}_{i} & 0 & -I_{n_{\mathrm{aw}}+n_{\mathrm{c}}+m} & 0 \\
\mathbb{G}_{i} & 0 & 0 & 0 & -I_{m}
\end{array}\right]\right)<0 .
\end{aligned}
$$


By using the Finsler's Lemma (Skelton et al. 1997), and noting that

$$
\left[\begin{array}{ccccc}
\mathbb{J}_{i} \mathbb{C} & 0 & -I_{n_{\mathrm{aw}}+n_{\mathrm{c}}+m} & 0 & 0 \\
0 & \mathbb{L}_{i} & 0 & -I_{n_{\mathrm{aw}}+n_{\mathrm{c}}+m} & 0 \\
\mathbb{G}_{i} & 0 & 0 & 0 & -I_{m}
\end{array}\right]^{\perp}=\left[\begin{array}{cc}
I_{n_{\mathrm{aw}}+n} & 0 \\
0 & I_{m} \\
\mathbb{J}_{i} \mathbb{C} & 0 \\
0 & \mathbb{L}_{i} \\
\mathbb{G}_{i} & 0
\end{array}\right]
$$

one gets

$$
\begin{aligned}
& {\left[\begin{array}{cc}
I_{n_{\text {aw }}+n} & 0 \\
0 & I_{m} \\
\mathbb{J}_{i} \mathbb{C} & 0 \\
0 & \mathbb{L}_{i} \\
\mathbb{G}_{i} & 0
\end{array}\right]^{\prime}\left(\left[\begin{array}{c}
\mathbb{A}_{i}^{\prime} \\
\mathbb{B}_{i}^{\prime} \\
\mathbb{T}_{i}^{\prime} \\
\mathbb{T}_{i}^{\prime} \\
0
\end{array}\right] \mathbb{P}_{j}\left[\begin{array}{c}
\mathbb{A}_{i}^{\prime} \\
\mathbb{B}_{i}^{\prime} \\
\mathbb{T}_{i}^{\prime} \\
\mathbb{T}_{i}^{\prime} \\
0
\end{array}\right]^{\prime}-\left[\begin{array}{c}
I_{\hat{n}} \\
0 \\
0 \\
0 \\
0
\end{array}\right] \mathbb{P}_{i}\left[\begin{array}{c}
I_{\hat{n}} \\
0 \\
0 \\
0 \\
0
\end{array}\right]^{\prime}-\operatorname{He}\left(\left[\begin{array}{c}
0 \\
I_{m} \\
0 \\
0 \\
0
\end{array}\right] \mathbb{U}_{i}\left[\begin{array}{c}
\mathbb{K}_{i}^{\prime} \\
I_{m} \\
\mathbb{F}^{\prime} \\
\mathbb{F}^{\prime} \\
I_{m}
\end{array}\right]\right)\right)\left[\begin{array}{cc}
I_{n_{\mathrm{aw}}+n} & 0 \\
0 & I_{m} \\
\mathbb{J}_{i} \mathbb{C} & 0 \\
0 & \mathbb{L}_{i} \\
\mathbb{G}_{i} & 0
\end{array}\right]} \\
& =\left[\begin{array}{c}
\left(\mathbb{A}_{i}+\mathbb{T}_{i} \mathbb{J}_{i} \mathbb{C}\right)^{\prime} \\
\left(\mathbb{B}_{i}+\mathbb{T}_{i} \mathbb{L}_{i}\right)^{\prime}
\end{array}\right] \mathbb{P}_{j}\left[\begin{array}{c}
\left(\mathbb{A}_{i}+\mathbb{T}_{i} \mathbb{J}_{i} \mathbb{C}\right)^{\prime} \\
\left(\mathbb{B}_{i}+\mathbb{T}_{i} \mathbb{L}_{i}\right)^{\prime}
\end{array}\right]^{\prime}-\left[\begin{array}{cc}
\mathbb{P}_{i}\left(\mathbb{G}_{i}+\mathbb{K}_{i}+\mathbb{F} \mathbb{J}_{i} \mathbb{C}\right)^{\prime} \mathbb{U}_{i} \\
\star & 2 \mathbb{U}_{i}+\operatorname{He}\left(\mathbb{U}_{i} \mathbb{F} \mathbb{L}_{i}\right)
\end{array}\right]<0 .
\end{aligned}
$$

Pre- and post-multiplying the inequality $(57)$ by $\left(\eta^{\prime}(t) \Psi^{\prime}\left(v_{\mathrm{c}}(t)\right)\right) \neq 0$ and its transpose and by identifying $i=\sigma(t)$ and $j=\sigma(t+1)$, it follows

$$
\mathbb{V}(\eta(t+1), \sigma(t+1))-\mathbb{V}(\eta(t), \sigma(t))-2 \Psi^{\prime}\left(v_{\mathrm{c}}(t)\right) \mathbb{U}_{i}\left[\Psi\left(v_{\mathrm{c}}(t)\right)+v_{\mathrm{c}}(t)+\mathbb{G}_{i} \eta(t)\right]<0 .
$$

The LMI (54) can be rewritten, by applying the change of variables $\mathbb{G}_{i}=\mathbb{X}_{i}^{-1} \mathbb{Q}_{i}$, as

$$
\left[\begin{array}{ccc}
\mathbb{P}_{i} & 0_{\hat{n} \times 1} & 0_{\hat{n} \times m} \\
0_{1 \times \hat{n}} & \rho_{(\ell)}^{2} & I_{m,(\ell)} \\
0_{m \times \hat{n}} & I_{m,(\ell)}^{\prime} & 0_{n}
\end{array}\right]+\operatorname{He}\left(\left[\begin{array}{c}
0_{\hat{n} \times m} \\
0_{1 \times m} \\
-\mathbb{X}_{i}
\end{array}\right]\left[\mathbb{G}_{i} 0_{m \times 1}-I_{m}\right]\right)>0 .
$$

Due to the relation

$$
\left[\mathbb{G}_{i} 0_{m \times 1}-I_{m}\right]^{\perp}=\left[\begin{array}{cc}
I_{\hat{n}} & 0 \\
0 & 1 \\
\mathbb{G}_{i} & 0_{m \times 1}
\end{array}\right]=\left[\begin{array}{c}
I_{\hat{n}+1} \\
{\left[\mathbb{G}_{i} 0_{m \times 1}\right]}
\end{array}\right]
$$

the Finsler's lemma allows to verify the inequality

$$
\begin{aligned}
{\left[\begin{array}{c}
I_{\hat{n}+1} \\
{\left[\mathbb{G}_{i} 0_{m \times 1}\right.}
\end{array}\right]^{\prime}\left[\begin{array}{ccc}
\mathbb{P}_{i} & 0_{\hat{n} \times 1} & 0_{\hat{n} \times m} \\
0_{1 \times \hat{n}} & \rho_{(\ell)}^{2} & I_{m,(\ell)} \\
0_{m \times \hat{n}} & I_{m,(\ell)}^{\prime} & 0_{n}
\end{array}\right] } & {\left[\begin{array}{c}
I_{\hat{n}+1} \\
{\left[\mathbb{G}_{i} 0_{m \times 1}\right]}
\end{array}\right] } \\
& =\left[\begin{array}{cc}
\mathbb{P}_{i} & \star \\
\left(\mathbb{G}_{i}\right)_{(\ell)} & \rho_{(\ell)}^{2}
\end{array}\right]>0_{\hat{n}+1}, \quad \forall(i, \ell) \in \mathcal{I} \times\{1, \cdots, m\} .
\end{aligned}
$$

Applying a Schur complement on the inequality (61), we have:

$$
\mathbb{P}_{i} \geq \frac{1}{\rho_{(\ell)}^{2}}\left(\mathbb{G}_{i}\right)_{(\ell)}^{\prime}\left(\mathbb{G}_{i}\right)_{(\ell)},
$$

which implies that $L_{\mathbb{V}_{\max }}$ is included in $\mathcal{S}\left(\left\{\mathbb{G}_{i}\right\}_{i \in \mathcal{I}}, \rho\right)$. Due to Lemma 4.1 , the cone bounded sector condition (49) is thus verified. The exponential stability is then ensured in $L_{\mathbb{V}_{\max }}$, which 
ends the proof.

Theorem 4.2 providing a stabilizing solution inside $L_{\mathbb{V}_{\max }}$, a solution to Problem 2.6 is given by the following optimization problem, aiming at maximizing the estimate $L_{\mathbb{V}_{\max }}$ of the basin of attraction.

Optimization problem 4.3

$$
\begin{gathered}
\min _{\mathbb{P}_{i}, \mathbb{X}_{i}, \mathbb{Q}_{i}, \mathbb{Y}_{i}, \mathbb{U}_{i}, \mathbb{Z}_{i}, \mathbb{M}_{i}, \mathbb{N}_{i}, \beta} \beta \\
\text { subject to }(32)-(33) \text { and } \\
\beta I_{\hat{n}}-\mathbb{P}_{i}>0, \quad \forall i \in \mathcal{I} .
\end{gathered}
$$

Remark 6: It should be pointed out that the result proposed in Theorem 4.2 is based on Finsler's Lemma and a convenient linearization by a change of variables. In the case where the LMIs (53) and (54) are not feasible, an additional degree of freedom may be introduced by modifying the multiplier appearing in inequality (55). Actually, the multiplier

$$
\left[\begin{array}{ccc}
0 & 0 & 0 \\
0 & 0 & 0 \\
\mathbb{M}_{i} & 0 & 0 \\
0 & \mathbb{N}_{i} & 0 \\
0 & 0 & \mathbb{X}_{i}
\end{array}\right]
$$

may be replaced by other ones, such as

$$
\left[\begin{array}{ccc}
0 & 0 & 0 \\
0 & 0 & 0 \\
2 \mathbb{M}_{i} & 0 & 0 \\
\mathbb{M}_{i} & \mathbb{N}_{i} & \mathbb{X}_{i} \\
0 & 0 & 2 \mathbb{X}_{i}
\end{array}\right]
$$

In this modification, the only one constraint allowing the change of variables linearizing the inequalities is to respect that the columns should be factorized at right respectively by $\mathbb{M}_{i}, \mathbb{N}_{i}$ and $\mathbb{X}_{i}$. That means that the multiplier can be expressed as the product: $\operatorname{Mdiag}\left(\mathbb{M}_{i} ; \mathbb{N}_{i} ; \mathbb{X}_{i}\right), M$ being any constant matrix of appropriate dimensions.

\section{Joint static anti-windup controller and min-switching law designs}

This section is devoted to solving Problem 2.7. First of all, some preliminaries related to the Metzler matrices are given. More details may be found in (Geromel and Colaneri 2006a,b). A matrix is called Metzler, if its off diagonal coefficients are nonnegative. The particular set $\mathcal{M}_{d}$ is defined as

$$
\mathcal{M}_{\mathrm{d}}=\left\{\Pi \in \mathbb{R}^{N \times N}, \pi_{j i} \geq 0, \forall(i, j) \in \mathcal{I}^{2}, \sum_{j \in \mathcal{I}} \pi_{j i}=1, \forall i \in \mathcal{I}\right\}
$$

For $\Pi \in \mathcal{M}_{\mathrm{d}}$ and a set of positive definite symmetric matrices $P_{i},(i \in \mathcal{I})$, we use the notation $P_{p, i}=\sum_{j \in \mathcal{I}} \pi_{j i} P_{j}$.

By considering the switched quadratic function $V$ defined by (28), the Lyapunov function can 
be built

$$
V_{\text {min }}:\left\{\begin{array}{l}
\mathbb{R}^{n} \longrightarrow \mathbb{R}^{+}, \\
\xi \longmapsto V_{\min }=\min _{i \in \mathcal{I}} V(\xi, i) .
\end{array}\right.
$$

Similarly to Section 3, the unitary level set of the Lyapunov function (67) is defined by

$$
L_{V_{\min }}=\left\{\xi \in \mathbb{R}^{n} ; V_{\min }(\xi) \leq 1\right\} .
$$

The level set $L_{V_{\min }}$ could be reformulated as indicated in the following lemma, (Hu 2007).

Lemma 5.1: The level set $L_{V_{\min }}$ defined by (68) verifies

$$
L_{V_{\text {min }}}=\bigcup_{i \in \mathcal{I}} \mathcal{E}\left(P_{i}\right)
$$

Proof $\xi \in L_{V_{\min }}$ is equivalent to the existence of $i_{0} \in \mathcal{I}$ such that $V\left(\xi, i_{0}\right) \leq 1$, that is $\xi \in \mathcal{E}\left(P_{i_{0}}\right)$, which is equivalent to $\xi \in \bigcup_{i \in \mathcal{I}} \mathcal{E}\left(P_{i}\right)$.

Let us define the set $\mathcal{T}_{i}$ associated with the mode $i \in \mathcal{I}$. For a given set of matrices $H_{i} \in \mathbb{R}^{m \times n}$,

$$
\mathcal{T}_{i}\left(H_{i}, \rho\right)=\left\{\xi \in \mathbb{R}^{n} ;-\rho \leq H_{i} \xi \leq \rho\right\}
$$

Lemma 5.2: Consider matrices $G_{i} \in \mathbb{R}^{m \times n}$. If the vector $\xi$ is an element of $\mathcal{T}_{i}\left(G_{i}, \rho\right)$, then the sector condition

$$
\Psi^{\prime}\left(v_{c}\right) U_{i}\left[\operatorname{sat}\left(v_{c}\right)+G_{i} \xi\right] \leq 0
$$

is verified for the mode $i \in \mathcal{I}$ and for any diagonal positive definite matrix $U_{i} \in \mathbb{R}^{m \times m}$.

Remark 7: It should be emphasized that the relation (71) in Lemma 5.2 is only ensured for the mode $i$ indexing the set $\mathcal{T}_{i}$ and not necessarily for the other ones. Furthermore, the link between Lemmas 3.1 and 5.2 may be established by noting that $\mathcal{S}\left(\left\{H_{i}\right\}_{i \in \mathcal{I}}, \rho\right)=\cap_{i \in \mathcal{I}} \mathcal{T}_{i}\left(H_{i}, \rho\right)$.

The following theorem provides sufficient conditions for the local joint controller and switching law stabilization of the system (23).

Theorem 5.3: Assuming there exist, for any $(i, j) \in \mathcal{I}^{2}$, matrices $Z_{i} \in \mathbb{R}^{\left(m+n_{c}\right) \times m}, Y_{i} \in$ $\mathbb{R}^{m \times n}, J_{i} \in \mathbb{R}^{n \times n}$, symmetric positive definite matrices $\Upsilon_{i j} \in \mathbb{R}^{n \times n}$ and $W_{i} \in \mathbb{R}^{n \times n}$ and diagonal positive definite matrices $S_{i} \in \mathbb{R}^{m \times m}$ and a Metzler matrix $\Pi \in \mathcal{M}_{d}$ satisfying the bilinear inequalities

$$
\left[\begin{array}{ccc}
J_{i}+J_{i}^{\prime}-\sum_{j \in \mathcal{I}} \pi_{j i} \Upsilon_{i j} & \star & \star \\
W_{i} \boldsymbol{A}_{i}^{\prime} & W_{i} & \star \\
S_{i} \boldsymbol{B}_{i}^{\prime}+Z_{i}^{\prime} \boldsymbol{R}_{i}^{\prime} & \left(\boldsymbol{K}_{i} W_{i}+Y_{i}\right) & \left(2 S_{i}+H e\left(\boldsymbol{F} Z_{i}\right)\right)
\end{array}\right]>0, \quad \forall i \in \mathcal{I},
$$

and the linear ones

$$
\left[\begin{array}{cc}
\Upsilon_{i j} & \star \\
J_{i} & W_{j}
\end{array}\right]>0, \quad \forall(i, j) \in \mathcal{I}^{2}
$$

and finally the LMIs (33), then the joint anti-windup controller consisting of $\boldsymbol{E}_{a w, i}=Z_{i} S_{i}^{-1}$ and 
the switching law

$$
\sigma(t)=\arg \min _{i \in \mathcal{I}} V(\xi(t), i)
$$

locally exponentially stabilize the system (23) in the set $L_{V_{\min }}$, with $P_{i}=W_{i}^{-1}$.

Proof By applying the Schur complement on the inequality (73), we have $\Upsilon_{i j}-J_{i}^{\prime} W_{j}^{-1} J_{i}>0$. This implies, with $P_{i}=W_{i}^{-1}$, that

$$
J_{i}+J_{i}^{\prime}-\sum_{j \in \mathcal{I}} \pi_{j i} \Upsilon_{i j}<J_{i}+J_{i}^{\prime}-J_{i}^{\prime}\left(\sum_{j \in \mathcal{I}} \pi_{j i} W_{j}^{-1}\right) J_{i}<\left(\sum_{j \in \mathcal{I}} \pi_{j i} W_{j}^{-1}\right)^{-1}=P_{p, i}^{-1}
$$

due to the property $J+J^{\prime}-J^{\prime} M J-M^{-1}=\left(J-M^{-1}\right)^{\prime} M\left(J-M^{-1}\right) \geq 0$ for $M=M^{\prime}>0$. The inequality (75) combined with the inequalities (72) leads to

$$
\left[\begin{array}{ccc}
P_{p, i}^{-1} & \star & \star \\
W_{i}^{\prime} \boldsymbol{A}_{i}^{\prime} & W_{i} & \star \\
\left(S_{i} \boldsymbol{B}_{i}^{\prime}+Z_{i}^{\prime} \boldsymbol{R}_{i}^{\prime}\right) & \left(\boldsymbol{K}_{i} W_{i}+Y_{i}\right) & \left(2 S_{i}+\operatorname{He}\left(\boldsymbol{F} Z_{i}\right)\right)
\end{array}\right]>0
$$

By identifying $U_{i}=S_{i}^{-1} ; G_{i}=Y_{i} W_{i}^{-1}$ and pre- and post-multiplying the inequality (76) by $\operatorname{diag}\left(P_{p, i} ; P_{i} ; U_{i}\right)$ and its transpose leads to

$$
\left[\begin{array}{ccc}
P_{p, i} & \star & \star \\
\boldsymbol{A}_{i}^{\prime} P_{p, i} & P_{i} & \star \\
\left(\boldsymbol{B}_{i}^{\prime}+U_{i} Z_{i}^{\prime} \boldsymbol{R}_{i}^{\prime}\right) P_{p, i} & U_{i}\left(\boldsymbol{K}_{i}+G_{i}\right) & \left(2 U_{i}+\operatorname{He}\left(U_{i} \boldsymbol{F} Z_{i} U_{i}\right)\right)
\end{array}\right]>0
$$

Identifying $\boldsymbol{E}_{\mathrm{aw}, i}=Z_{i} S_{i}^{-1}=Z_{i} U_{i}$ and using a Schur complement yields

$$
\left[\begin{array}{cc}
P_{i} & \star \\
U_{i}\left(\boldsymbol{K}_{i}+G_{i}\right) & \left(2 U_{i}+\operatorname{He}\left(U_{i} \boldsymbol{F} \boldsymbol{E}_{\mathrm{aw}, i}\right)\right)
\end{array}\right]-\left[\begin{array}{c}
\boldsymbol{A}_{i}^{\prime} \\
\left(\boldsymbol{B}_{i}+\boldsymbol{R}_{i} \boldsymbol{E}_{\mathrm{aw}, i}\right)^{\prime}
\end{array}\right] P_{p, i}\left[\boldsymbol{A}_{i}\left(\boldsymbol{B}_{i}+\boldsymbol{R}_{i} \boldsymbol{E}_{\mathrm{aw}, i}\right)\right]>0 .
$$

Let us prove now that the inequalities (78) ensure that the function $V_{\min }$ is a Lyapunov function for the closed-loop system (23)-(24) with the switching law (74). The function $V_{\text {min }}$ defined by (67) is a minimum over a finite set of values, it has then the following properties:

- $V_{\min }(\xi) \geq 0, \forall \xi \in \mathbb{R}^{n}$,

- $V_{\min }(\xi)=0 \Rightarrow \xi=0$,

- $V_{\min }(\cdot)$ is radially unbounded.

In order to prove that $V_{\min }$ is a Lyapunov function, it suffices to ensure that $V_{\min }(\xi(t+1))-$ $V_{\min }(\xi(t))<0$, along the closed-loop trajectories. Let us denote $z(t)=\left(\begin{array}{c}\xi(t) \\ \Psi\left(v_{\mathrm{c}}(t)\right)\end{array}\right)$. By noting $\Omega \subset \mathbb{R}^{N}$ the unit simplex of dimension $N$, we have because each column of a Metzler matrix 
$\Pi \in \mathcal{M}_{\mathrm{d}}$ belongs to $\Omega$,

$$
\begin{aligned}
& V_{\min }(\xi(t+1))=\min _{j \in \mathcal{I}} \xi^{\prime}(t+1) P_{j} \xi(t+1),=\min _{j \in \mathcal{I}} z(t)^{\prime}\left[\begin{array}{c}
\boldsymbol{A}_{i}^{\prime} \\
\left(\boldsymbol{B}_{i}+\boldsymbol{R}_{i} \boldsymbol{E}_{\mathrm{aw}, i}\right)^{\prime}
\end{array}\right] P_{j}\left[\boldsymbol{A}_{i}\left(\boldsymbol{B}_{i}+\boldsymbol{R}_{i} \boldsymbol{E}_{\mathrm{aw}, i}\right)\right] z(t) \\
&=\min _{\lambda \in \Omega} z(t)^{\prime} {\left[\begin{array}{c}
\boldsymbol{A}_{i}^{\prime} \\
\left(\boldsymbol{B}_{i}+\boldsymbol{R}_{i} \boldsymbol{E}_{\mathrm{aw}, i}\right)^{\prime}
\end{array}\right] \sum_{j \in \mathcal{I}} \lambda_{j} P_{j}\left[\boldsymbol{A}_{i}\left(\boldsymbol{B}_{i}+\boldsymbol{R}_{i} \boldsymbol{E}_{\mathrm{aw}, i}\right)\right] z(t) } \\
& \leq z(t)^{\prime}\left[\begin{array}{c}
\boldsymbol{A}_{i}^{\prime} \\
\left(\boldsymbol{B}_{i}+\boldsymbol{R}_{i} \boldsymbol{E}_{\mathrm{aw}, i}\right)^{\prime}
\end{array}\right] \sum_{j \in \mathcal{I}} \pi_{j i} P_{j}\left[\boldsymbol{A}_{i}\left(\boldsymbol{B}_{i}+\boldsymbol{R}_{i} \boldsymbol{E}_{\mathrm{aw}, i}\right)\right] z(t) .
\end{aligned}
$$

Due to the inequality (78), it follows by identifying $i=\sigma(t)$,

$$
V_{\min }(\xi(t+1)) \leq z(t)^{\prime}\left[\begin{array}{cc}
P_{i} & \star \\
\left(U_{i}\left(\boldsymbol{K}_{i}+G_{i}\right)\right) & \left(2 U_{i}+\operatorname{He}\left(U_{i} \boldsymbol{F} \boldsymbol{E}_{\mathrm{aw}, i}\right)\right)
\end{array}\right] z(t),
$$

that is

$$
V_{\min }(\xi(t+1))-V_{\min }(\xi(t))<2 \Psi^{\prime}\left(v_{\mathrm{c}}(t)\right) U_{i}\left[\Psi\left(v_{\mathrm{c}}(t)\right)+v_{\mathrm{c}}(t)+G_{i} \xi(t)\right] .
$$

In order to prove the decreasing of the function $V_{\min }$ along the closed-loop trajectories, from the inequality (81), the sector condition (71) has to be satisfied for the mode defined by (74). This is done by inequality (33) allowing $\mathcal{E}\left(P_{i}\right) \subset \mathcal{T}_{i}\left(G_{i}\right)$. Applying the switching law (74) implies the local exponential stability in $L_{V_{\min }}$.

The estimate $L_{V_{\min }}$ of the basin of attraction being an union of ellipsoids, a way to increase its size is to increase the size of all the ellipsoids. This approach is considered in the following optimization problem providing a solution to Problem 2.7.

Optimization problem 5.4

$$
\begin{aligned}
& \min _{W_{i}, Y_{i}, Z_{i}, S_{i}, J_{i}, \Upsilon_{i j}, \Pi, \beta_{i}} \sum_{j \in \mathcal{I}} \beta_{j} \\
& \text { subject to (72)-(73)-(33) and } \\
& {\left[\begin{array}{cc}
\beta_{i} I_{n} & I_{n} \\
I_{n} & W_{i}
\end{array}\right]>0_{2 n}, \quad \forall i \in \mathcal{I} .}
\end{aligned}
$$

Remark 8: This is noteworthy that Optimization Problem 5.4 is not straightforwardly solvable due to the bilinear nature of inequalities (72), issued from the product of variables $\pi_{j i}$ and $\Upsilon_{i j}$. When the matrix $\Pi \in \mathcal{M}_{\mathrm{d}}$ is fixed, all the constraints of Optimization Problem 5.4 are LMIs. A suitable way, presented in (Geromel and Colaneri 2006a), consists in considering the Metzler matrix to belong into a parametrized class of matrices and to apply a line search procedure.

Even if it is more conservative and necessitates more inequalities, another way is proposed in (Geromel and Colaneri 2006a) by replacing BMIs (72) by sufficient LMIs

$$
\left[\begin{array}{ccc}
J_{i}+J_{i}^{\prime}-\left(\gamma \Upsilon_{i i}+(1-\gamma) \Upsilon_{i j}\right) & \star & \star \\
W_{i} \boldsymbol{A}_{i}^{\prime} & W_{i} & \star \\
S_{i} \boldsymbol{B}_{i}^{\prime}+Z_{i}^{\prime} \boldsymbol{R}_{i}^{\prime} & \boldsymbol{K}_{i} W_{i}+Y_{i} & \left(2 S_{i}+\operatorname{He}\left(\boldsymbol{F} Z_{i}\right)\right)
\end{array}\right]>0, \quad \forall(i, j) \in \mathcal{I}^{2},
$$

with a given scalar $\gamma$ such that $0 \leq \gamma<1$. A solution of inequalities (72) are then obtained by considering a matrix $\Pi \in \mathcal{M}_{\mathrm{d}}$, with $\pi_{i i}=\gamma$ and $\sum_{j \neq i, j \in \mathcal{I}} \pi_{j i}=(1-\gamma)$ and finally weighting 
inequalities (83) by $\pi_{j i}$ and summing the resulting inequalities over $j \in \mathcal{I}, j \neq i$.

It should be mentioned that the switching law designed by (74) requires the knowledge of the extended state $\xi(t)$ composed of the state $x(t)$ of the system and the state $x_{\mathrm{c}}(t)$ of the predefined controller. It could be more suitable to perform a switching law design based only on the state $x_{\mathrm{c}}(t)$ to avoid the knowledge of the state $x(t)$, as it has been underlined in (Geromel et al. 2008, Deaecto et al. 2010). That is important generally to implement the joint control laws in practice. Such a result can be obtained by imposing a particular structure to the matrices $P_{i}$ as follows.

$$
P_{i}=\left[\begin{array}{cc}
\Delta & \star \\
\Theta & \Xi_{i}
\end{array}\right], \quad i \in \mathcal{I}
$$

with $\Theta$ invertible, which induces a reformulation of the switching law design (74)

$$
\sigma(t)=\arg \min _{i \in \mathcal{I}} \xi(t)^{\prime} P_{i} \xi(t)=\arg \min _{i \in \mathcal{I}} x_{\mathrm{c}}(t)^{\prime} \Xi_{i} x_{\mathrm{c}}(t)
$$

Theorem 5.3 does not involve the variable $P_{i}$, but its inverse $W_{i}=P_{i}^{-1}$. The difficulty is thus to handle the particular structure of $P_{i}$ given by (84). A technique presented in (Geromel et al. 2008, Deaecto et al. 2010) allows it by elegant manipulations. Nevertheless this is based on a change of basis proposed in (Scherer et al. 1997) closely related to the joint design of a dynamic output feedback controller. This is not relevant for our framework, due to the fact that the dynamic output feedback controller is predefined. The next theorem provides an alternative of Theorem 5.3 taking as variables the matrices $P_{i}$ and allowing to set the structure (84).

Theorem 5.5: Assuming there exist, for any $i \in \mathcal{I}$, matrices $Z_{i} \in \mathbb{R}^{\left(m+n_{c}\right) \times m}, G_{i} \in \mathbb{R}^{m \times n}$, $M_{i} \in \mathbb{R}^{n \times n}, H_{i} \in \mathbb{R}^{n \times n}$, symmetric positive definite matrices $Q_{i} \in \mathbb{R}^{n \times n}$ and $P_{i} \in \mathbb{R}^{n \times n}$ and diagonal positive definite matrices $S_{i} \in \mathbb{R}^{m \times m}$ and a Metzler matrix $\Pi \in \mathcal{M}_{d}$ satisfying the bilinear inequalities

$$
\begin{gathered}
{\left[\begin{array}{ccc}
Q_{i} & \star & \star \\
\boldsymbol{A}_{i}^{\prime} & P_{i} & \star \\
\left(S_{i} \boldsymbol{B}_{i}^{\prime}+Z_{i}^{\prime} \boldsymbol{R}_{i}^{\prime}\right) & \left(\boldsymbol{K}_{i}+G_{i} 2 S_{i}+H e\left(\boldsymbol{F} Z_{i}\right)\right)
\end{array}\right]>0, \quad \forall i \in \mathcal{I},} \\
{\left[\begin{array}{cc}
H_{i}+H_{i}^{\prime}-Q_{i} & \star \\
\left(I_{n}-H_{i}-M_{i}\right) & M_{i}+M_{i}^{\prime}-\sum_{j \in \mathcal{I}} \pi_{j i} P_{j}
\end{array}\right]>0, \quad \forall i \in \mathcal{I},}
\end{gathered}
$$

and finally the LMIs (33), then the joint anti-windup controller consisting of $\boldsymbol{E}_{a w, i}=Z_{i} S_{i}^{-1}$ and the switching law (85) exponentially locally stabilize the system (23) in the set $L_{V_{\text {min }}}$.

Proof From inequalities (87), matrices $M_{i}$ are invertible. By applying the change of variables $N_{i}=H_{i} M_{i}^{-1}$, inequalities (87) can be reformulated as

$$
\left[\begin{array}{cc}
-Q_{i} & \star \\
I_{n} & -\sum_{j \in \mathcal{I}} \pi_{j i} P_{j}
\end{array}\right]+\operatorname{He}\left(\left[\begin{array}{c}
M_{i} \\
-M_{i}
\end{array}\right]\left[N_{i}-I_{n}\right]\right)>0, \quad \forall i \in \mathcal{I} .
$$


By noting that $\left[N_{i}-I_{n}\right]^{\perp}=\left[\begin{array}{c}I_{n} \\ N_{i}\end{array}\right]$, Finsler's Lemma yields

$$
\left[\begin{array}{l}
I_{n} \\
N_{i}
\end{array}\right]^{\prime}\left[\begin{array}{cc}
-Q_{i} & \star \\
I_{n} & -\sum_{j \in \mathcal{I}} \pi_{j i} P_{j}
\end{array}\right]\left[\begin{array}{l}
I_{n} \\
N_{i}
\end{array}\right]=-Q_{i}+N_{i}+N_{i}^{\prime}-N_{i}^{\prime}\left(\sum_{j \in \mathcal{I}} \pi_{j i} P_{j}\right) N_{i}>0
$$

which implies

$$
Q_{i}<\left(\sum_{j \in \mathcal{I}} \pi_{j i} P_{j}\right)^{-1}=P_{p, i}^{-1}
$$

Injecting these last inequalities into (86) recovers inequalities (77). The end of the proof is then the same as the one of Theorem 5.3 by noting that one replaces inequalities (33) with

$$
\left[\begin{array}{cc}
P_{i} & \star \\
G_{i,(\ell)} & \rho_{(\ell)}^{2}
\end{array}\right]>0, \quad \forall(i, \ell) \in \mathcal{I} \times\{1, \cdots, m\}
$$

and inequalities (40) with its Schur complement (41).

\section{Joint dynamic output anti-windup controller and min-switching law designs}

This section copes with solving Problem 2.8. The closed-loop system is given by equations (42) and (43), with the notation presented in Section 4. The joint design of a stabilizing switching law and a dynamic output feedback has been already proposed in the literature, see for instance (Geromel et al. 2008, Deaecto et al. 2010). As mentioned above, the point of view in these papers is to impose a structure on the switched Lyapunov matrices via their inverse. Here, in order to follow the choices in Section 5, taking as a variable the switched Lyapunov matrices, thanks to the use of Finsler's Lemma, we propose Theorem 6.1 to solve Problem 2.8.

Theorem 6.1: If there exist a Metzler matrix $\Pi \in \mathcal{M}_{d}$, a set of symmetric positive definite matrices $\mathbb{P}_{i} \in \mathbb{R}^{\hat{n} \times \hat{n}}$, matrices $\mathbb{Q}_{i} \in \mathbb{R}^{m \times \hat{n}}, \mathbb{X}_{i} \in \mathbb{R}^{m \times m}, \mathbb{Y}_{i} \in \mathbb{R}^{\left(n_{\text {aw }}+n_{c}+m\right) \times n_{\text {aw }}}, \mathbb{Z}_{i} \in \mathbb{R}^{\left(n_{\text {aw }}+n_{c}+m\right) \times m}$, $\mathbb{M}_{i} \in \mathbb{R}^{\left(n_{a w}+n_{c}+m\right) \times\left(n_{a w}+n_{c}+m\right)}$ and $\mathbb{N}_{i} \in \mathbb{R}^{\left(n_{a w}+n_{c}+m\right) \times\left(n_{a w}+n_{c}+m\right)}$, diagonal positive definite matrices $\mathbb{U}_{i} \in \mathbb{R}^{m \times m},(i \in \mathcal{I})$ such that, $\forall(i, j) \in \mathcal{I}^{2}$,

$$
\begin{aligned}
& {\left[\begin{array}{c}
\mathbb{A}_{i}^{\prime} \\
\mathbb{B}_{i}^{\prime} \\
\mathbb{T}_{i}^{\prime} \\
\mathbb{T}_{i}^{\prime} \\
0
\end{array}\right]\left(\sum_{j \in \mathcal{I}} \pi_{j i} \mathbb{P}_{j}\right)\left[\begin{array}{c}
\mathbb{A}_{i}^{\prime} \\
\mathbb{B}_{i}^{\prime} \\
\mathbb{T}_{i}^{\prime} \\
\mathbb{T}_{i}^{\prime} \\
0
\end{array}\right]^{\prime}-\left[\begin{array}{c}
I_{\hat{n}} \\
0 \\
0 \\
0 \\
0
\end{array}\right] \mathbb{P}_{i}\left[\begin{array}{c}
I_{\hat{n}} \\
0 \\
0 \\
0 \\
0
\end{array}\right]^{\prime}-H e\left(\left[\begin{array}{c}
0 \\
I_{m} \\
0 \\
0 \\
0
\end{array}\right] \mathbb{U}_{i}\left[\begin{array}{c}
\mathbb{K}_{i}^{\prime} \\
I_{m} \\
\mathbb{F}^{\prime} \\
\mathbb{F}^{\prime} \\
I_{m}
\end{array}\right]^{\prime}\right)} \\
& +H e\left(\left[\begin{array}{ccccc}
0 & 0 & 0 & 0 & 0 \\
0 & 0 & 0 & 0 & 0 \\
\mathbb{Y}_{i} \mathbb{C} & 0 & -\mathbb{M}_{i} & 0 & 0 \\
0 & \mathbb{Z}_{i} & 0 & -\mathbb{N}_{i} & 0 \\
\mathbb{Q}_{i} & 0 & 0 & 0 & -\mathbb{X}_{i}
\end{array}\right]\right)<0
\end{aligned}
$$


and inequalities (54) are satisfied, then the anti-windup dynamic output feedback defined by $\mathbb{L}_{i}=$ $\mathbb{N}_{i}^{-1} \mathbb{Z}_{i}$ and $\mathbb{J}_{i}=\mathbb{M}_{i}^{-1} \mathbb{Y}_{i},(i \in \mathcal{I})$ and the switching control law

$$
\sigma(t)=\arg \min _{i \in \mathcal{I}} \eta(t)^{\prime} \mathbb{P}_{i} \eta(t),
$$

(locally) exponentially stabilize the system (42) in the level set $L_{\mathbb{V}_{\min }}$.

Proof The proof is closely related to the one of Theorem 4.2 , where $\mathbb{P}_{j}$ has been replaced by $\left(\sum_{j \in \mathcal{I}} \pi_{j i} \mathbb{P}_{j}\right)$ to obtain, with $\mathbb{G}_{i}=\mathbb{X}_{i}^{-1} \mathbb{Q}_{i}$

$$
\mathbb{V}_{\min }(\xi(t+1))-\mathbb{V}_{\min }(\xi(t))<2 \Psi^{\prime}\left(v_{\mathrm{c}}(t)\right) \mathbb{U}_{i}\left[\Psi\left(v_{\mathrm{c}}(t)\right)+v_{\mathrm{c}}(t)+\mathbb{G}_{i} \xi(t)\right]
$$

It is then the same as that one of Theorem 5.3 in what concerns the estimate $L_{\mathbb{V}_{\min }}$ of the basin of attraction.

\section{$7 \quad$ Numerical results}

This section is devoted to illustrate the results obtained in the paper in order to emphasize their effectiveness and also their conservatism.

Example 7.1 Let us consider the result of Section 3 related to Problem 2.5. The switched system, extending the numerical example borrowed from (Gomes da Silva Jr. and Tarbouriech 2006, Example 1), is defined by $n_{\mathrm{p}}=n_{\mathrm{c}}=1, N=2, \rho=1$ and

$$
A_{1}=1.2 ; A_{2}=1.5 ; B_{1}=B_{2}=1 ; C_{1}=1 ; C_{2}=0.5 \text {. }
$$

The dynamic output stabilizing controller (3)-(4) is given by

$$
\begin{gathered}
A_{\mathrm{c}, 1}=1 ; \quad A_{\mathrm{c}, 2}=0.5 ; \quad B_{\mathrm{c}, 1}=-0.05 ; B_{\mathrm{c}, 2}=-0.01 ; \\
C_{\mathrm{c}, 1}=1 ; C_{\mathrm{c}, 2}=0.2 ; \quad D_{\mathrm{c}, 1}=-1 ; D_{\mathrm{c}, 2}=-1 .
\end{gathered}
$$

The system is not stable in open loop, but is stabilized by the dynamic output controller. One has $\boldsymbol{A}_{1}=\left[\begin{array}{cc}0.2 & 1 \\ -0.05 & 1\end{array}\right]$ and $\boldsymbol{A}_{2}=\left[\begin{array}{cc}1 & 0.2 \\ -0.005 & 0.5\end{array}\right]$. The set of matrices $\left\{\boldsymbol{A}_{1}, \boldsymbol{A}_{2}\right\}$ leads to an asymptotically stable difference inclusion $\xi(t+1) \in\left\{\boldsymbol{A}_{i} \xi(t), \forall i \in \mathcal{I}\right\}$. In order to emphasize this fact, let us consider the switching quadratic Lyapunov function defined by the matrices $\left[\begin{array}{cc}2.4292 & -1.9098 \\ -1.9098 & 26.4035\end{array}\right]>0_{2}$ and $\left[\begin{array}{cc}4.7888 & 1.7021 \\ 1.7021 & 11.5376\end{array}\right]>0_{2}$, for the first and second modes, respectively.

Optimization Problem 3.4, related to Theorem 3.3 leads to $\beta=1.3318$ and

$$
\begin{aligned}
& P_{1}=\left[\begin{array}{cc}
0.2342 & -0.1439 \\
-0.1439 & 1.3130
\end{array}\right] ; \quad P_{2}=\left[\begin{array}{cc}
0.2764 & 0.0737 \\
0.0737 & 0.5642
\end{array}\right] ; \\
& \boldsymbol{E}_{\mathrm{aw}, 1}=\left[\begin{array}{c}
1.1487 e-6 \\
-1+3.5 e-6
\end{array}\right] ; \quad \boldsymbol{E}_{\mathrm{aw}, 2}=\left[\begin{array}{c}
4.4380 e-7 \\
-1+8.83 e-8
\end{array}\right]
\end{aligned}
$$

The gains defined in (96) are called in the following, strategy a. The well posedness of the relation (24) is ensured thanks to Remark $\mathbf{3}$, because here $m=1$ and the scalars $1,\left(I_{m}+\boldsymbol{F} \boldsymbol{E}_{\text {aw, }, 1}\right)$ 
and $\left(I_{m}+\boldsymbol{F} \boldsymbol{E}_{\text {aw }, 2}\right)$ are invertible. The results are depicted in Figure 1. First of all, a comparison with the analysis of local stability when there is no anti-windup compensator is presented. When

$$
\boldsymbol{E}_{\mathrm{aw}, i}=0, \quad \forall i \in \mathcal{I},
$$

which is called in the following strategy $b$, the ellipsoids and the ball $\mathcal{E}\left(\beta I_{2}\right)$ are plotted in dashed line. It is noteworthy that in the case without anti-windup, in addition of the origin, there are two (unstable) equilibrium points for the second mode: $\xi_{e} \pm\left(\begin{array}{c}-2 \\ 0.02\end{array}\right)$. By applying Optimization Problem 3.4, each mode has two equilibrium points, in plus of the origin: for the first mode with the computed $\boldsymbol{E}_{a w, 1}$, one gets $\xi_{e}= \pm\left(\begin{array}{c}5 \\ 3.2373\end{array}\right)$; for the second mode with computed $\boldsymbol{E}_{a w, 2}$, one gets $\xi_{e}= \pm\left(\begin{array}{c}-2 \\ 0.0066\end{array}\right)$. The equilibrium points without and with anti-windup are depicted by squares and crosses in Figure 1, respectively. Note also that the ellipsoids $\mathcal{E}\left(P_{i}\right)$ obtained with anti-windup are bigger ( $\beta$ has been reduced by $18 \%$ ), as shown in Figure 1.

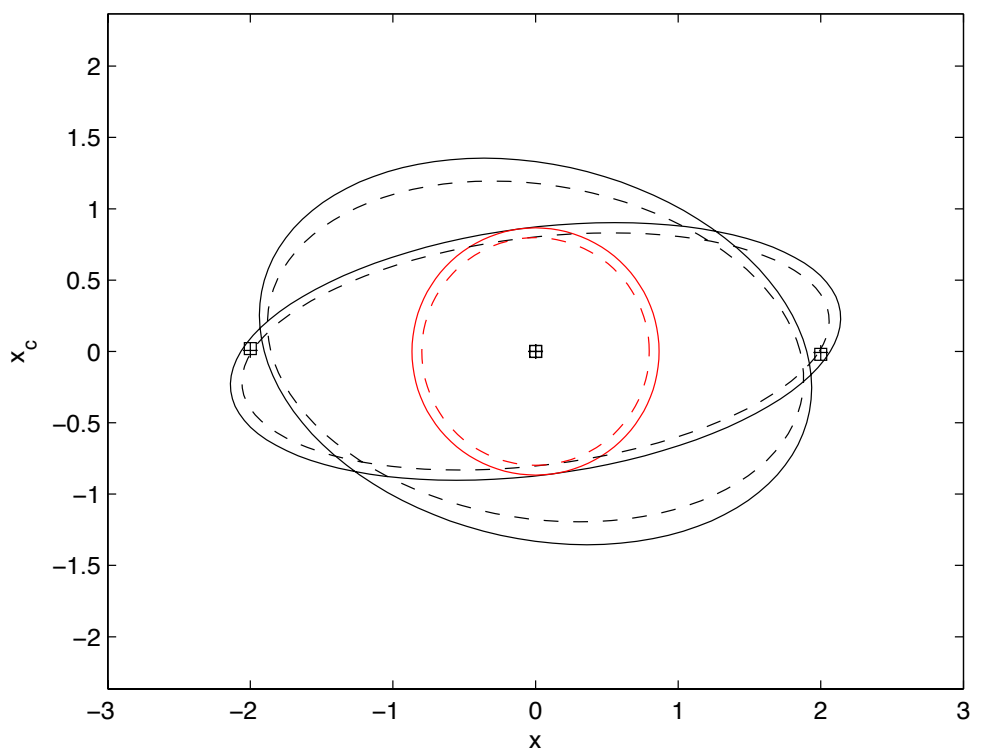

Figure 1. Example 7.1 - Ellipsoids $\mathcal{E}\left(P_{1}\right)$ and $\mathcal{E}\left(P_{2}\right)$ in black solid line associated with strategy a; ellipsoids related to the basin of attraction without antiwindup controller $\left(E_{\mathrm{aw}, \mathrm{i}}=0\right.$ or strategy $\left.b\right)$ in black dashed line and the associated balls $\mathcal{E}\left(\beta I_{2}\right)$ of the two cases, respectively in red solid and red dashed line. The squares and crosses denote equilibrium points for strategies $a$ and $b$.

It is interesting to investigate the fact that the terms $\left(I_{m}+\boldsymbol{F} \boldsymbol{E}_{\mathrm{aw}, i}\right), i \in \mathcal{I}$ are invertible but numerically close to singularity. In order to avoid such a numerical difficulty, we can impose a structure to the matrices $\boldsymbol{E}_{\mathrm{aw}, i}$, or roughly speaking to the matrices $Z_{i}$ in LMI (32) in Theorem 3.3. When imposing $Z_{i}=\left[\begin{array}{c}\tilde{Z}_{i} \\ 0_{m}\end{array}\right]$, with $\tilde{Z}_{i} \in \mathbb{R}^{n_{\mathrm{c}} \times m}$, we obtain $\beta=1.3319$ and

$$
\begin{aligned}
& P_{1}=\left[\begin{array}{cc}
0.2342 & -0.1439 \\
-0.1439 & 1.3130
\end{array}\right] ; \quad P_{2}=\left[\begin{array}{cc}
0.2764 & 0.0737 \\
0.0737 & 0.5642
\end{array}\right] ; \\
& \boldsymbol{E}_{\mathrm{aw}, 1}=\left[\begin{array}{c}
0.3277 \\
0
\end{array}\right] ; \boldsymbol{E}_{\mathrm{aw}, 2}=\left[\begin{array}{c}
5.0174 \\
0
\end{array}\right] .
\end{aligned}
$$


The gains defined by (99) are associated with strategy $c$ in the following. The numerical results in what concerns $P_{1}$ and $P_{2}$ by Equation (98) are the same as the ones given by Equation (95) for the considered numerical precision. The value of $\beta$ has been modified less than $0.01 \%$. Nevertheless $\boldsymbol{E}_{\text {aw,1 }}$ and $\boldsymbol{E}_{\text {aw,2 }}$ have been changed. The equilibrium points have not been modified.

To deepen the illustration, Figure 2 is proposed: the sets $\mathcal{S}\left(\left\{\boldsymbol{K}_{i}\right\}_{i \in \mathcal{I}}, \rho\right)$ and $\mathcal{S}\left(\left\{G_{i}\right\}_{i \in \mathcal{I}}, \rho\right)$ are plotted in addition to the ellipsoids $\mathcal{E}\left(P_{i}\right), i \in \mathcal{I}$ and the ball $\mathcal{E}\left(\beta I_{2}\right)$. In accordance with the proposed approach, the ellipsoids are not inside the area of linearity $\left(\mathcal{S}\left(\left\{\boldsymbol{K}_{i}\right\}_{i \in \mathcal{I}}, \rho\right)\right)$ but are included in the modal area where the sector conditions are verified. In addition, due to the optimization scheme, these ellipsoids reach the bounds of the validity area of the sector conditions. Two families of trajectories are proposed in Figure 2. Two initial states are considered (plotted with o) $\xi(0)=\xi_{1}=\left(\begin{array}{c}2 \\ 0.35\end{array}\right)$ and $\xi(0)=\xi_{2}=\left(\begin{array}{c}1.926 \\ -0.16\end{array}\right)$. Starting from $\xi_{2}$, two trajectories, associated with the switching rules $\{1 ; 1 ; 1 ; 1 ; \cdots\}$ and $\{2 ; 1 ; 2 ; 1 ; 2 ; 2 ; 1 ; \cdots\}$ are shown: they are stable. Nevertheless starting from $\xi_{1}$, which is outside of $L_{V_{\max }}$, the trajectory generated by the switching rule $\{2 ; 1 ; 2 ; 1 ; 2 ; 2 ; 1 ; \cdots\}$ is unstable and the one induced by the switching rule $\{2 ; 2 ; 2 ; 2 ; \cdots\}$ is stable.

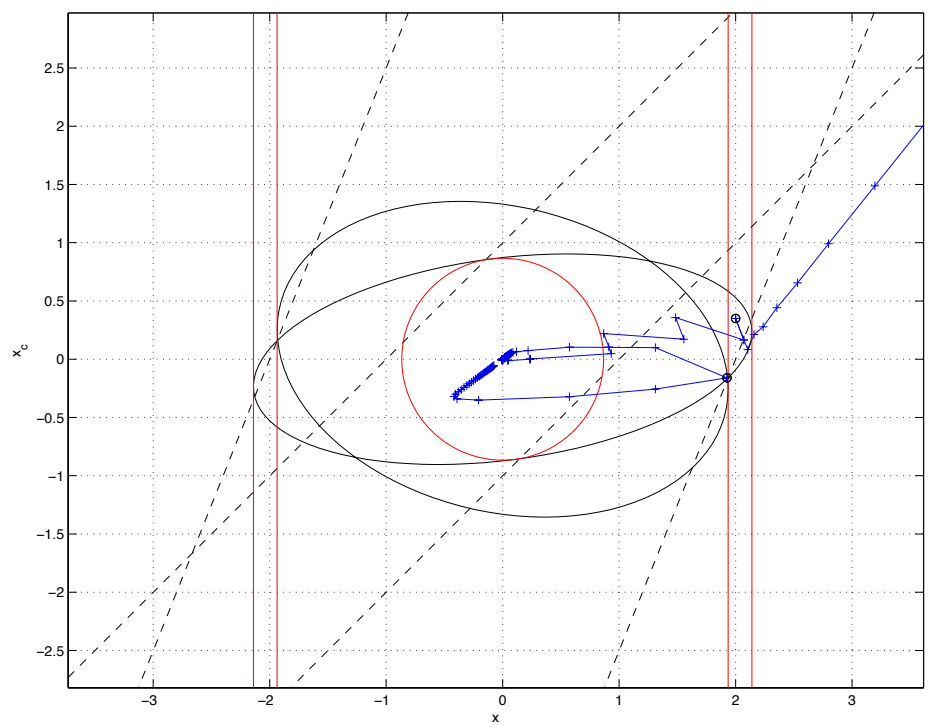

Figure 2. Example $7.1-\mathcal{S}\left(\left\{G_{i}\right\}_{i \in \mathcal{I}}, \rho\right)$ in red solid line. $\mathcal{S}\left(\left\{\boldsymbol{K}_{i}\right\}_{i \in \mathcal{I}}, \rho\right)$ in black dashed line. Ellipsoids $\mathcal{E}\left(P_{i}\right), i \in \mathcal{I}$ in black solid lines and the ball $\mathcal{E}\left(\beta I_{2}\right)$ in red solid line. Two families of trajectories are also emphasized.

The control signals for each mode corresponding to the cases with anti-windup and without anti-windup are shown in Figure 3. The initial state considered is $\xi(0)=\xi_{2}=\left(\begin{array}{c}1.926 \\ -0.16\end{array}\right)$. A comparison of the control signal for the three strategies is depicted in Figure 4. One can observe quite different transient behaviors depending on the chosen strategy.

Example 7.2 Let consider now the proposed solution to Problem 2.7, via the same data as in Example 7.1. The system being defined by a set of matrices $\left\{\boldsymbol{A}_{1} ; \boldsymbol{A}_{2}\right\}$, which leads to an asymptotically stable difference inclusion $\xi(t+1) \in\left\{\boldsymbol{A}_{i} \xi(t), \forall i \in \mathcal{I}\right\}$, all the switching law stabilize the system without anti-windup controller (for instance the switching law activating always the same mode stabilizes the system). When $\boldsymbol{E}_{\text {aw }, i}=0$ is imposed, that is without antiwindup controller, we obtain the results depicted in Figure 5. In this case, there exist three equilibrium points. The origin is stable but the equilibria $\xi_{e}=\left(\begin{array}{c}-2 \\ 0.2\end{array}\right)$ and $\xi_{e}=-\left(\begin{array}{c}-2 \\ 0.2\end{array}\right)$ are unstable and are associated with mode 2. In Figure 5, we can see that these unstable equilibrium points belong to the conic state-partition activating mode 1 . These are no equilibrium points for the system driven by the switching law. Nevertheless they limit the ellipsoid related to mode 2 . 

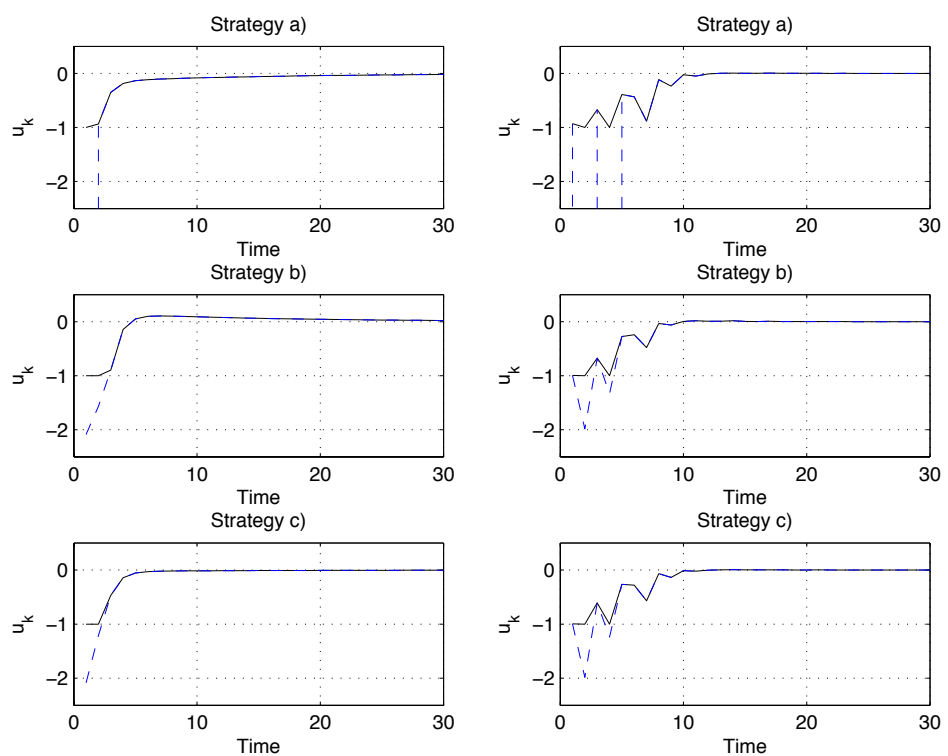

Figure 3. Example 7.1 - Time-evolution of the control signal, and the saturated control signal for strategies $a, b$ and $c$, on the left for the switching rule $\{1 ; 1 ; 1 ; 1 ; \cdots\}$ and on the right for the switching rule $\{2 ; 1 ; 2 ; 1 ; 2 ; 2 ; 1 ; \cdots\}$.
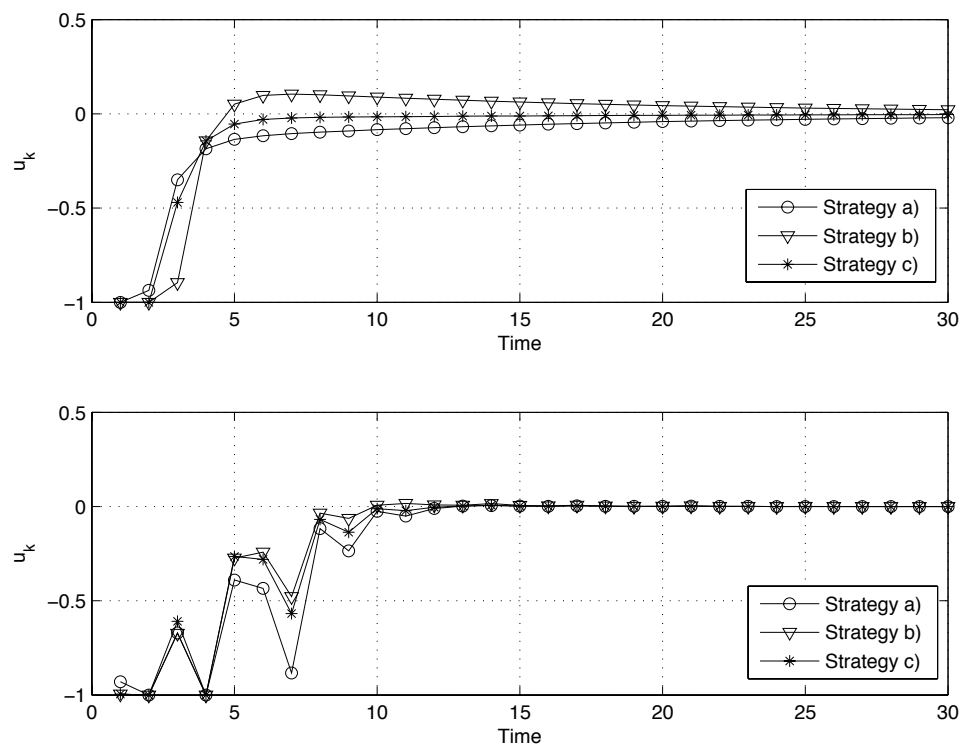

Figure 4. Example 7.1 - Comparison of the saturated control signal for strategies $a, b$ and $c$, on the top for the switching rule $\{1 ; 1 ; 1 ; 1 ; \cdots\}$ and at the bottom for the switching rule $\{2 ; 1 ; 2 ; 1 ; 2 ; 2 ; 1 ; \cdots\}$.

Several trajectories are plotted to attempt to illustrate the basin of attraction. When the antiwindup gain $\boldsymbol{E}_{\text {aw }, i}$ is not null but computed from our approach, we have the results depicted in Figure 6 . There are five equilibrium points. The origin is stable and common to the two modes. The equilibrium points for mode 2 are $\xi_{e}= \pm\left(\begin{array}{c}-2 \\ 0.065\end{array}\right)$. They belong to the activation area of mode 1 and are not equilibrium points for the closed-loop system. The equilibrium points for mode 1 are $\xi_{e}= \pm\left(\begin{array}{c}-5 \\ 0.0636\end{array}\right)$ and are in the activation area of this mode. They are equilibrium points for the closed-loop system. Introducing an anti-windup static gain allows only an improvement of 
the cost function to minimize of $3 \%$. This limited improvement may be explained by the presence of the equilibrium point of mode 2 restricting the ellipsoid of mode 2.

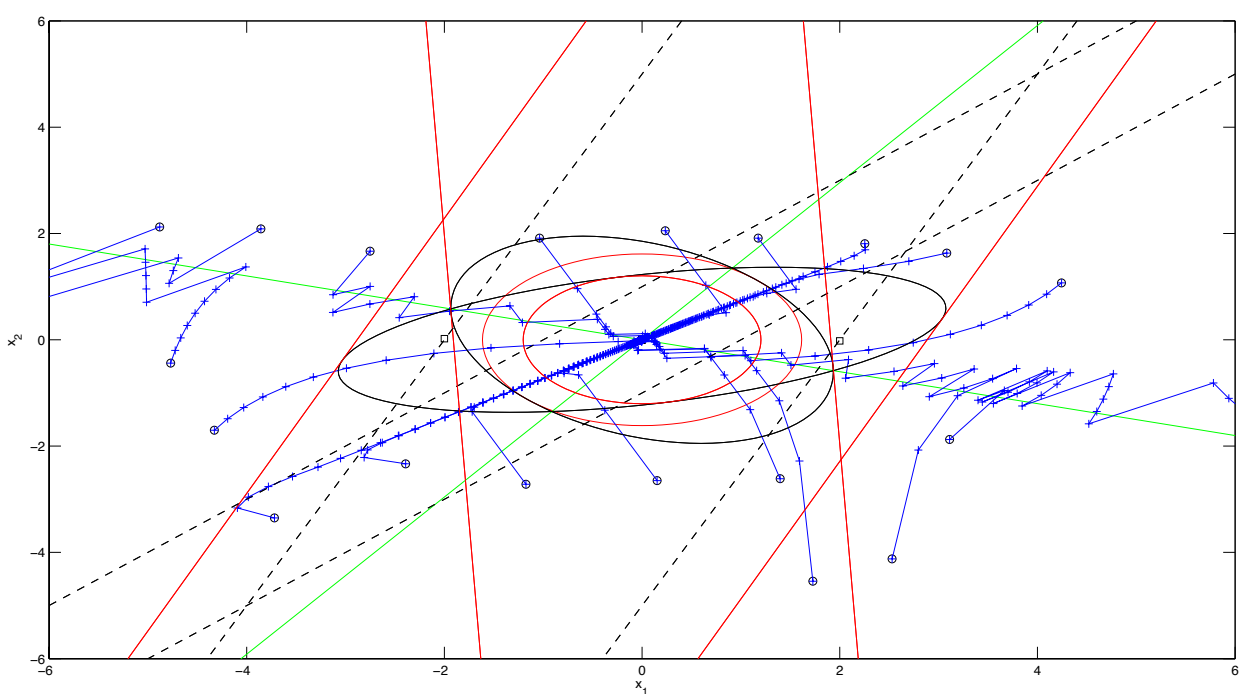

Figure 5. Example $7.2-\mathcal{S}\left(\left\{G_{i}\right\}_{i \in \mathcal{I}}, \rho\right)$ in red solid line. $\mathcal{S}\left(\left\{\boldsymbol{K}_{i}\right\}_{i \in \mathcal{I}}, \rho\right)$ in black dashed line. Conic state-partition in green solid line. Ellipsoids $\mathcal{E}\left(P_{i}\right), i \in \mathcal{I}$ in black solid line and the balls $\mathcal{E}\left(\beta_{i} I_{2}\right)$ in red solid line. Equilibrium points with squares.

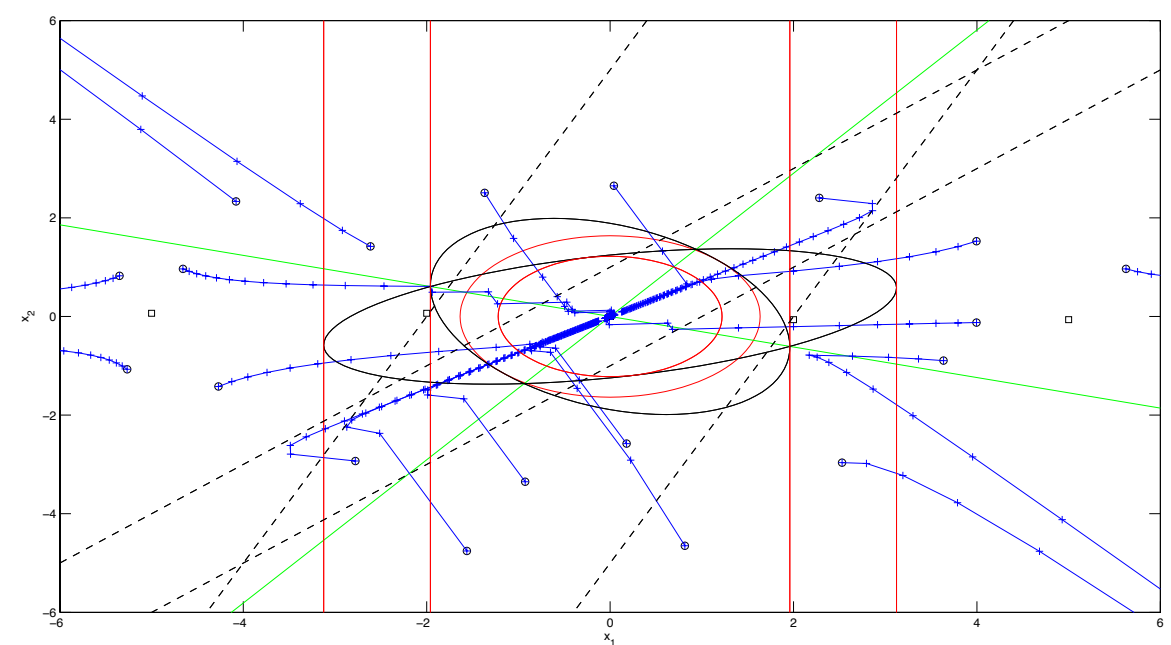

Figure 6. Example $7.2-\mathcal{S}\left(\left\{G_{i}\right\}_{i \in \mathcal{I}}, \rho\right)$ in red solid line. $\mathcal{S}\left(\left\{\boldsymbol{K}_{i}\right\}_{i \in \mathcal{I}}, \rho\right)$ in black dashed line. Conic state-partition in green solid line. Ellipsoids $\mathcal{E}\left(P_{i}\right), i \in \mathcal{I}$ in black solid line and the balls $\mathcal{E}\left(\beta_{i} I_{2}\right)$ in red solid line. Equilibrium points with squares.

The example is thus modified in order to illustrate the behavior of the proposed solution when the difference inclusion $\xi(t+1) \in\left\{\boldsymbol{A}_{i} \xi(t), \forall i \in \mathcal{I}\right\}$ is not stable. Only the dynamic output controller is modified by considering

$$
\begin{gathered}
A_{\mathrm{c}, 1}=1.1 ; \quad A_{\mathrm{c}, 2}=-0.5 ; \quad B_{\mathrm{c}, 1}=-0.05 ; B_{\mathrm{c}, 2}=-0.01 ; \\
C_{\mathrm{c}, 1}=1 ; C_{\mathrm{c}, 2}=0.5 ; \quad D_{\mathrm{c}, 1}=-1 ; \quad D_{\mathrm{c}, 2}=-0.9 .
\end{gathered}
$$


One has then

$$
\boldsymbol{A}_{1}=\left[\begin{array}{cc}
0.2 & 1 \\
-0.05 & 1.1
\end{array}\right] ; \quad \boldsymbol{A}_{2}=\left[\begin{array}{cc}
1.05 & 0.5 \\
-0.005 & -0.5
\end{array}\right]
$$

which are not Schur. The improvement of the cost function $\beta_{1}+\beta_{2}$ by considering the controller with the anti-windup loop is $92 \%$ with respect to the case without the anti-windup loop.

Example 7.3 Consider the system (7)-(10) described by the following data borrowed from (Zhang and Zhao 2012):

$$
\begin{gathered}
A_{1}=\left[\begin{array}{cc}
1 & -0.3 \\
1 & 0
\end{array}\right] ; A_{2}=\left[\begin{array}{cc}
0.806 & 0.2418 \\
0.806 & 0
\end{array}\right] ; \\
B_{1}=\left[\begin{array}{c}
0.48 \\
-0.4
\end{array}\right] ; B_{2}=\left[\begin{array}{c}
0.13 \\
-1.3
\end{array}\right] ; \\
C_{1}=\left[\begin{array}{l}
0.3450 .69
\end{array}\right] ; C_{2}=[0.17-0.3] \\
A_{c, 1}=\left[\begin{array}{c}
0.1133-0.016 \\
0.0138-0.1143
\end{array}\right] ; A_{c, 2}=\left[\begin{array}{c}
-0.0515-0.1398 \\
0.0043-0.0309
\end{array}\right] ; \\
B_{c, 1}=\left[\begin{array}{l}
-0.0209 \\
-0.0904
\end{array}\right] ; B_{c, 2}=\left[\begin{array}{c}
-0.0525 \\
0.0286
\end{array}\right] ; \\
C_{c, 1}=[2.3191-0.4768] ; C_{c 2}=[-2.9468-1.5688] ; \\
D_{c, 1}=-0.5437 ; D_{c, 2}=-1.5199 ;
\end{gathered}
$$

By applying the Optimization Problem 3.4, related to Theorem 3.3, one obtains

$$
\boldsymbol{E}_{a w, 1}=\left[\begin{array}{c}
0.14 e-4 \\
0.16 e-4 \\
-1+6.7 e-5
\end{array}\right] ; \boldsymbol{E}_{a w, 2}=\left[\begin{array}{c}
0.65 e-5 \\
0.60 e-5 \\
-1+9.9 e-5
\end{array}\right] ; \beta=3.2 e-4
$$

We compare also with the static anti-windup given in (Zhang and Zhao 2012):

$$
\boldsymbol{E}_{a w, 1}=\left[\begin{array}{c}
0.0074 \\
0.0056 \\
0
\end{array}\right] ; \boldsymbol{E}_{a w, 2}=\left[\begin{array}{c}
-0.0590 \\
0.0654 \\
0
\end{array}\right]
$$

by using Theorem 3.3 and the Optimization Problem 3.4 reformulated in an analysis point of view.

Table 1 shows the results obtained with static and dynamic anti-windup loops, with the static anti-windup given in (Zhang and Zhao 2012) and without anti-windup.

Then, one can observe in Table 1 that our technique allows to largely improve the size of the region of stability for the closed-loop system: for example, in the static case, thanks the anti-windup loop, $\beta$ has been reduced by $99.8 \%$; in the dynamic case, one drastically reduces $\beta$. 


\begin{tabular}{|c|c|}
\hline Type of AW & $\beta$ \\
\hline \hline no AW $\left(\boldsymbol{E}_{a w, i}=0\right)$ & 0.17 \\
\hline static AW given in (Zhang and Zhao 2012) & 0.15 \\
\hline static AW obtained from Optimization Problem 3.4 & $3.2 \mathrm{e}-4$ \\
\hline dynamic AW obtained from Optimization Problem 4.3 & $1.04 \mathrm{e}-11$ \\
\hline
\end{tabular}

Table 1. Example 7.3 - Results of the optimization problem for different cases.

\section{Conclusion}

The anti-windup strategies for discrete-time switched systems subject to input saturation has been investigated in this paper. Both static and dynamic anti-windup controllers have been designed with the objective to maximize the estimate of the basin of attraction. The results have been considered in two frameworks relative to the assumption about the switching law: on one hand the switching law is assumed to be arbitrary and on the other hand the switching law is assumed to be a part of the control law. Optimization problems have been formulated under LMI conditions to design the (joint) controllers. Illustrations are given to show the efficiency of these approaches.

The technique proposed paves the way for future research in several directions. Hence, it could be interesting to perform the design of anti-windup controller by considering the model recovery anti-windup scheme as in (Galeani et al. 2009, Zaccarian and Teel 2011). Furthermore, the problem of enlarging the region of stability for the closed-loop system could be completed by imposing some performance requirement. Finally, one could benefit from the use of more general Lyapunov functions (for example by adapting those used in (Gonzaga et al. 2012b)) to reduce the conservatism of estimation of the region of stability.

\section{Acknowledgment}

This work was partially supported by ANR project ArHyCo, Programme " Systèmes Embarqués et Grandes Infrastructures" - ARPEGE, contract number ANR-2008 SEGI 004 01-30011459, by ANR COMPACS -"Computation Aware Control Systems", ANR-13-BS03-004 and by the European Community's Seventh Framework Programme (FP7/2007-2013) under grant agreement $\mathrm{n}^{\circ}$ 257462: HYCON2 Network of Excellence "Highly-Complex and Networked Control Systems" and finally by Région Lorraine.

\section{References}

Agrachev, A.A., and Liberzon, D. (2001), "Lie-algebraic stability criteria for switched systems," SIAM J. Control Optim., 40, 253-270.

Benzaouia, A., Saturated switching systems, Vol. 426 of Lecture Notes in Control and Information Sciences, Springer (2012).

Boyd, S., El Ghaoui, L., Feron, E., and Balakrishnan, V., Linear Matrix Inequalities in System and Control Theory, SIAM Studies in Applied Mathematics (1994).

Branicky, M.S. (1998), "Multiple Lyapunov functions and other analysis tools for switched and hybrid systems.," IEEE Transactions on Automatic Control, 43, 475-482.

Bruckner, M., Galeani, S., and Del Re, L. (2010), "Switching adaptative realization of a weakened anti-windup compensator," in Proceedings of the 49th IEEE Conference on Decision and Control, Atlanta, GA, USA, pp. 5233-5238.

Bruckner, M., Galeani, S., and Del Re, L. (2013), "Robustified Anti-Windup via Switching Adaptation," IEEE Transactions on Automatic Control, 58, 731-737. 
Cao, Y.Y., and Lin, Z. (2003), "Stability analysis of discrete-time systems with actuator saturation by a saturation-dependent Lyapunov function," Automatica, 39, 1235-1241.

Chen, Y., Fei, S., Zhang, K., and Fu, Z. (2012), "Control Synthesis of Discrete-Time Switched Linear Systems with Input Saturation Based on Minimum Dwell Time Approach," Circuits Syst. ES Signal Process, 31, 779-795.

Daafouz, J., and Bernussou, J. (2002), "Robust Dynamic Output Feedback Control for Switched Systems," in Proceedings of the 41st IEEE Conference on Decision and Control, December, Las Vegas, Nevada USA, pp. 4389-4394.

Daafouz, J., Millérioux, G., and Iung, C. (2002), "A Poly-Quadratic stability based approach for linear switched systems," International Journal of Control, 75, 1302-1310.

Daafouz, J., Riedinger, P., and Iung, C. (2002), "Stability Analysis and Control Synthesis for Switched Systems : A Switched Lyapunov Function Approach," IEEE Transactions on Automatic Control, 47, 1883-1887.

Daafouz, J., Riedinger, P., and Iung, C. (2003), "Observer-based switched control design with pole placement for discrete-time switched systems," International Journal of Hybrid systems, $3,263-281$.

Daafouz, J., Tarbouriech, S., and Sigalotti, M. (eds.) Hybrid Systems with Constraints, WileyISTE (2013).

Daimond, P., and Opoitsev, V.I. (2001), "Stability of Linear Difference and Differential Inclusions," Automation and Remote Control, 62, 695-703.

Deaecto, G.S., Geromel, J.C., and Daafouz, J. (2010), "Full order dynamic output feedback $\mathcal{H}_{\infty}$ control design for discrete-time switched linear systems," in proceedings of the 18th Mediterranean Conference on Control \&5 Automation, Marrakech, Morocco, pp. 1212-1217.

Duan, C., and Wu, F. (2012), "Output feedback control for switched linear systems subject to actuator saturation," International Journal of Control, 85, 1532-1545.

Duan, C., and Wu, F. (2014), "New results on switched linear systems with actuator saturation," International Journal of Systems Science, online.

Durieu, C., Polyak, B., and Walter, E. (1996), "Trace versus determinant in ellipsoidal approximation with application to state estimation," in Proceedings of the 13th Triennial IFAC World Congress, Vol. 1, San Francisco, pp. 43-48.

Galeani, S., Tarbouriech, S., Turner, M., and Zaccarian, L. (2009), "A Tutorial on Modern AntiWindup Design," European Journal of Control, 15, 418-440.

Geromel, J.C., Bolzern, P., and Colaneri, P. (2008), "Dynamic Output Feedback Control of Switched Linear Systems," IEEE Transactions on Automatic Control, 53, 720-733.

Geromel, J.C., and Colaneri, P. (2006b), "Stability and stabilization of continuous-time switched linear systems," SIAM J. Control Optim., 45, 1915-1930.

Geromel, J.C., and Colaneri, P. (2006a), "Stability and stabilization of discrete-time switched systems," International Journal of Control, 79, 719-728.

Goebel, R., Sanfelice, R.G., and Teel, A.R., Hybrid Dynamical Systems: Modeling, Stability, and Robustness, Princeton University Press (2012).

Gomes da Silva Jr., J.M., Paim, C., and Castelan, E.B. (2001), "Stability and Stabilization of Linear Discrete-Time subject to Control Saturation," in 1st IFAC Symp. on System Structure and Control (SSSC'01), Prague, Czech Republic.

Gomes da Silva Jr., J.M., and Tarbouriech, S. (2006), "Anti-windup design with guaranteed regions of stability for discrete-time linear systems," Systems $\&$ Control Letters, pp. 184-192.

Gonzaga, C.A.C., Jungers, M., and Daafouz, J. (2012a), "Stability analysis and stabilisation of switched nonlinear systems," International Journal of Control, 85, 822-829.

Gonzaga, C.A.C., Jungers, M., and Daafouz, J. (2012b), "Stability analysis of discrete-time Lur'e systems.," Automatica, 48, 2277-2283.

Gonzaga, C.A.C., Jungers, M., Daafouz, J., and Castelan, E.B. (2011), "A new class of Lyapunov functions for nonstandard switching systems: the stability analysis problem," in 50th IEEE Conference on Decision and Control and European Control Conference (CDC-ECC'11), 
Orlando, Florida, USA, pp. 411-416.

Grimm, G., Teel, A.R., and Zaccarian, L. (2008), "The $L_{2}$ anti-windup problem for discrete-time linear systems: Definition and solutions," Systems $\&$ Control Letters, 57, 356-364.

Gurvits, L. (1995), "Stability of Discrete Linear Inclusion," Linear Algebra and its Applications, $231,47-85$.

Hante, F.M., and Sigalotti, M. (2011), "Converse Lyapunov theorems for switched systems in Banach and Hilbert spaces," SIAM J. Control Optim., 49, 752-770.

Herrmann, G., Turner, M.C., and Postlethwaite, I. (2003), "Discrete-time anti-windup: Part 2 Extension to the sampled-data case," in European Control Conference (ECC'03), Cambridge, United Kingdom.

Hu, T. (2007), "Switching Law Construction for Discrete-Time Systems Via Composite Quadratic Functions," in Proceedings of the 2007 American Control Conference, New York City, USA.

$\mathrm{Hu}, \mathrm{T}$. , and Lin, Z., Control systems with actuator saturation. Analysis and design, Birkhäuser (2001).

Hu, T., Teel, A.R., and Zaccarian, L. (2005), "Regional Anti-windup Compensation for Linear Systems with Input Saturation," in Proceeding in the American Control Conference, Portland, OR, USA, pp. 3397-3398.

$\mathrm{Hu}$, T., Teel, A.R., and Zaccarian, L. (2008), "Anti-windup synthesis for linear control systems with input saturation: Achieving regional, nonlinear performance," Automatica, 44, 512-519.

Johansson, M., Piecewise Linear Control Systems: A computational Approach, no. 284 in: Lecture Notes in Control and Information Sciences, Springer-Verlag (2002).

Jungers, M., Gonzaga, C.A.C., and Daafouz, J. (2012), "Min-Switching Stabilization for DiscreteTime Switching Systems with Nonlinear Modes," in 4th IFAC Conference on Analysis and Design of Hybrid Systems, ADHS 2012, Eindhoven, The Netherlands, pp. 234-239.

Jungers, M., Gonzaga, C.A.C., and Daafouz, J. (2013), "Min-Switching Local Stabilization for Discrete-Time Switching Systems with Nonlinear Modes," Nonlinear Analysis: Hybrid Systems, $9,18-26$.

Kapila, V., and Grigoriadis, K. (eds.) Actuator saturation control, Marcel Dekker, Inc. (2002).

Li, Y., and Lin, Z. (2013), "Design of Saturation-Based Switching Anti-Windup Gains for the Enlargement of the Domain of Attraction," IEEE Transactions on Automatic Control, 58, 1810-1816.

Liberzon, D., Switching in Systems and Control, Vol. in series Systems and Control: Foundations and Applications., Birkhäuser, Boston, MA (2003).

Liberzon, D., Hespanha, J.P., and Morse, A.S. (1999), "Stability of switched linear systems: A Lie-algebraic condition," Systems \&6 Control Letters, 37, 117-122.

Liberzon, D., and Morse, A. (1999), "Basic Problems in Stability and Design of Switched Systems," IEEE Control Systems Magazine, 19, 59-70.

Liu, X.L., and Duan, G.R. (2005), "Robust Stabilization of Switched Systems with Input Saturation," in Proceedings of the Fourth International Conference on Machine Learning and Cybernectics, Guangzhou, China, pp. 1021-1025.

Lu, L., and Lin, Z. (2009), "A Switching Anti-windup Design Using Multiple Lyapunov Functions," in Joint 48th IEEE Conference on Decision and Control and 28th Chinese Control Conference, December, Shangai, P.R. China, pp. 1920-1925.

Lu, L., and Lin, Z. (2010), "A Switching Anti-windup Design Using Multiple Lyapunov Functions," IEEE Transactions on Automatic Control, 55, 142-148.

Lunze, J., and Lamnabhi-Lagarrigue, F. (eds.) Handbook of Hybrid Systems Control: Theory, Tools, Applications, Cambridge (2009).

Ma, Y., Yang, G., and Guan, W. (2009), "Robust stabilization of switched discrete-time systems with actuator saturation," Journal of Control Theory and Applications, 7, 454-458.

Melin, J., Jungers, M., Daafouz, J., and Iung, C. (2011), "Performance Analysis and Design of Dynamic Output Feedback Control for Switched Systems," International Journal of Control, $84,253-260$. 
Molchanov, A.P., and Pyatnitskiy, Y.S. (1989), "Criteria of asymptotic stability of differential and difference inclusions encounterd in control theory," Systems \& Control Letters, 13, 59-64.

Monovich, T., and Margaliot, M. (2011), "Analysis of discrete-time linear switched systems: a variational approach," SIAM J. Control Optim., 49, 808-829.

Mulder, E.F., and Kothare, M.V. (2000), "Synthesis of Stabilizing Anti-Windup Controllers Using Piecewise Quadratic Lyapunov Functions," in Proceedings of the American Control Conference, Chicago, IL, USA, pp. 3239-3243.

Ni, W., and Cheng, D. (2010), "Control of switched linear systems with input saturation," International Journal of Systems Science, 41, 1057-1065.

Scherer, C., Gahinet, P., and Chilali, M. (1997), "Multiobjective Output-Feedback Control via LMI Optimization," IEEE Transactions on automatic control, 42, 896-911.

Schutter, B.D. (2000), "Optimal control of a class of linear hybrid systems with saturations," SIAM J. Control Optim., 39, 835-851.

Shorten, R., Corless, M., Middleton, R., Klinge, S., and Wulff, K. (2009), "A quadratic stability result for singular switched systems with application to anti-windup control," in Proceeding in the American Control Conference, St. Louis, MO, USA, pp. 1917-1922.

Shorten, R., Wirth, F., Mason, O., Wulff, K., and King, C. (2007), "Stability Criteria for Switched and Hybrid Systems," SIAM Review, 49, 545-592.

Skelton, R., Iwasaki, T., and Grigoriadis, K., A unified algebraic approach to linear control design, Taylor and Francis (1997).

Sun, Z., and Ge, S.S. (2005), "Analysis and synthesis of switched linear control systems," Automatica, 41, 181-195.

Sun, Z., and Ge, S.S., Stability Theory of Switched Dynamical Systems, Springer (2011).

Tarbouriech, S., Garcia, G., and Glattfelder, A. (eds.) Advanced strategies in control systems with input and output constraints, LNCIS vol.346 Springer Verlag. (2007).

Tarbouriech, S., Garcia, G., Gomes da Silva Jr., J.M., and Queinnec, I., Stability and Stabilization of Linear Systems with Saturating Actuators, Springer (2011).

Tarbouriech, S., Prieur, C., and Gomes da Silva Jr., J.M. (2006), "Stability analysis and stabilization of systems presenting nested saturations," IEEE Transactions on Automatic Control, $51,1364-1371$.

Tiwari, P.Y., Mulder, E.F., and Kothare, M.V. (2007), "Synthesis of Stabilizing Antiwindup Controllers Using Piecewise Quadratic Lyapunov Functions," IEEE Transactions on Automatic Control, 52, 2341-2345.

Tsitsiklis, J., and Blondel, V. (1997), "The Lyapunov exponent and joint spectral radius of pairs of matrices are hard - when not impossible - to compute and to approximate," Mathematics of Control, Signals, and Systems, 10, 31-40.

Turner, M.C., Herrmann, G., and Postlethwaite, I. (2003), "Discrete-time anti-windup: Part 1 - Stability and performance," in European Control Conference (ECC'03), Cambridge, United Kingdom.

Turner, M., and Zaccarian, L. (2006), "Special Issue: Anti-windup," International Journal of Systems Science, 37, 65-139.

van der Schaft, A., and Schumacher, H., An Introduction to Hybrid Dynamical Systems, SpringerVerlag, London (2000).

Ye, H., Michel, A.N., and Hou, L. (1998), "Stability theory for hybrid dynamical systems.," IEEE Trans. Autom. Control, 43, 461-474.

Yuan, C., and Wu, F. (2014), "Switching control of linear systems subject to asymmetric actuator saturation," International Journal of Control, 88, 204-215.

Zaccarian, L., and Teel, A.R., Modern anti-windup synthesis, Princeton University Press (2011).

Zhang, X., Wang, M., and Zhao, J. (2012), "Stability analysis and antiwindup design of uncertain discrete-time switched linear systems subject to actuator saturation," J Control Theory Appl, $10,325-331$.

Zhang, X., and Zhao, J. (2012), " $\mathcal{L}_{2}$-gain Analysis and Anti-windup Design of Discrete-time 
Switched Systems with Actuator Saturation," International Journal of Automation and Computing, 9, 369-377.

Zhang, X., Zhao, J., and Dimirovski, G.M. (2011), " $\mathcal{L}_{2}$-gain analysis and control synthesis of uncertain discrete-time switched linear systems with time delay and actuator saturation," International Journal of Control, 84, 1746-1758.

Zhang, X., Zhao, J., Dimirovski, G.M., and Ma, R. (2010), "Robust State Feedback Stabilization of Uncertain Switched Linear Systems Subject to Actuator Saturation," in Proceedings of the American Control Conference, Baltimore, MD, USA, pp. 3269-3274.

Zhao, X.Q., and Zhao, J. (2014), " $\mathcal{L}_{2}$-gain analysis and output feedback control for continuoustime switched systems with actuator saturation," Nonlinear Dynamics, 78, 1357-1367. 